\title{
Differential Effects of Oxytocin Receptor Antagonists, Atosiban and Nolasiban, on Oxytocin Receptor-Mediated Signaling in Human Amnion and Myometrium $\mathbf{s}$
}

\author{
Sung Hye Kim, Oliver Pohl, Andre Chollet, Jean-Pierre Gotteland, Adam D. J. Fairhurst, \\ Phillip R. Bennett, and Vasso Terzidou \\ Institute of Reproductive and Developmental Biology, Hammersmith Hospital Campus (S.H.K., A.D.J.F., P.R.B., V.T.) and \\ Chelsea and Westminster Hospital (V.T.), Imperial College London, London, United Kingdom; and ObsEva SA, Geneva, \\ Switzerland (O.P., A.C., J.-P.G.)
}

Received August 31, 2016; accepted January 30, 2017

\section{ABSTRACT}

One of the most established roles of oxytocin (OT) is in inducing uterine contractions and labor. Apart from inducing contractions, our recent studies showed that OT can also activate proinflammatory pathways in both human myometrial and amnion cells, which suggests that the proinflammatory role of OT should be taken into account when developing tocolytics targeting the OT/oxytocin receptor (OTR) system. The OTR antagonist, atosiban, is currently used therapeutically for the treatment of preterm labor. We previously showed that atosiban fails to inhibit the proinflammatory effects of OT in human amnion; atosiban alone activates nuclear factor $-\kappa \mathrm{B}(\mathrm{NF}-\kappa \mathrm{B})$ and mitogen activated protein kinases, thus upregulating downstream prolabor genes. In contrast with our findings with atosiban, the presence of the orally active OTR antagonist, nolasiban, reduced the effect of OT on NF- $\kappa$ B and p38 kinase activation in both myometrial and amnion cells. Consistent with the activation of these inflammatory mediators, OT led to increases in the expression of cyclooxygenase-2 and phosphorylated cytosolic phospholipase $A_{2}$, which was reflected in prostaglandin $\mathrm{E}_{2}$ synthesis. Inhibition of NF- $\kappa \mathrm{B}$ activation by nolasiban also translated to suppression of downstream prolabor gene expression, such as cyclooxygenase-2, C-C motif chemokine ligand 2, interleukin-6, and interleukin-8. We also demonstrated that nolasiban treatment alone has no significant stimulatory effect on both the myometrium and amnion. In conclusion, our findings indicate that nolasiban possesses promising potential as a novel tocolytic agent for both acute and maintenance therapy, as it inhibits both myometrial contractions and the proinflammatory effects of OT without the biased agonist effects.

\section{Introduction}

Complications arising from preterm labor account for $65 \%$ of neonatal deaths and $50 \%$ of neurologic disabilities worldwide. Advances in the care of preterm infants have dramatically increased rates of survival, which rise sharply from $1 \%$ at 23 weeks to $99 \%$ at 32 weeks. However, this is associated with a reciprocal increase in the risk of complications (Lawn et al., 2013). Although advances have been made in the prediction and prevention of preterm birth in women identified at high risk based on past history, the overall rate has not decreased.

This research was supported in part by the Genesis Research Trust [Grant P14758] and the National Institute for Health Research Biomedical Research Centre based at Imperial College Healthcare NHS Trust [Grant P45272] and Imperial College London. V.T. has worked as a consultant to GlaxoSmithKline. P.R.B. has worked as a consultant to Boehringer Ingelheim, GlaxoSmithKline, Merck Serono, ObsEva SA, and Tokyo Tanabe Pharmaceuticals, drug companies with an interest in the pharmacological effects of oxytocin and oxytocin antagonists. P.R.B. holds shares of ObsEva SA. O.P., A.C., and J.-P.G. are salaried employees of ObsEva SA.

dx.doi.org/10.1124/mol.116.106013.

S This article has supplemental material available at molpharm. aspetjournals.org
The 2012 World Health Organization report Born Too Soon (Howson et al., 2013) highlighted the need for effective tocolytic drugs to delay preterm birth in women with preterm contractions

Infection and/or inflammation is associated with activation of contractile pathways leading to both term and preterm labor. Furthermore, infection/inflammation and subsequent increases in proinflammatory cytokines were found to be one of the most potent causes of perinatal brain injury (Dommergues et al., 2000; Thornton et al., 2012). In normal birth weight infants at term, chorioamnionitis and intrauterine exposure to maternal infection are associated with periventricular leukomalacia and cerebral palsy (Grether and Nelson, 1997; Wu and Colford, 2000). In the case of established chorioamnionitis, it is possible that an effective tocolytic drug based solely on the inhibition of uterine contractions could result in prolonged exposure of the fetus to an adverse environment. Therefore, an optimal tocolytic should also have anti-inflammatory activity. Although the precise mechanisms responsible for initiating labor in humans remain ambiguous,

ABBREVIATIONS: AVP, arginine vasopressin; CCL, C-C motif chemokine ligand; COX, cyclooxygenase; DMEM, Dulbecco's modified Eagle's medium; DMSO, dimethylsulfoxide; ERK, extracellular signal-regulated kinase; GPCR, G protein-coupled receptor; ${ }^{1} \mathrm{H}$ NMR, proton nuclear magnetic resonance spectroscopy; IL, interleukin; MAPK, mitogen-activated protein kinase; NF- $\kappa \mathrm{B}$, nuclear factor- $\kappa \mathrm{B}$; OT, oxytocin; OTR, oxytocin receptor; PG, prostaglandin; $\mathrm{p}-\mathrm{CPLA}_{2}$, phosphorylated cytosolic phospholipase $\mathrm{A}_{2}$; TLC, thin layer chromatography. 
TABLE 1

Primer sequences for real-time polymerase chain reaction

\begin{tabular}{lll}
\hline Target Gene & \multicolumn{1}{c}{ Forward Primer Sequence (5' to $\left.3^{\prime}\right)$} & \multicolumn{1}{c}{ Reverse Primer Sequence (5' to $\left.3^{\prime}\right)$} \\
\hline L19 & GCGGAAGGGTACAGCCAAT & GCAGCCGGCGCAAA \\
GAPDH & TGATGACATCAAGAAGGTGGTGAAG & TCCTTGGAGGCCATGTAGGCCAT \\
CCL2 & TCTGTGCCTGCTGCTCATAG & AGATCTCCTTGGCCACAATG \\
CCL5 & CCATATTCCTCGGACACCAC & TGTACTCCCGAACCCATTTC \\
COX-2 & TGTGCAACACTTGAGTGGCT & ACTTTCTGTACTGCGGGTGG \\
IL-6 & CCTTCCAAAGATGGCTGAAA & AGCTCTGGCTTGTTCCTCAC \\
IL-8 & GCCTTCCTGATTTCTGCAGC & CGCAGTGTGGTCCACTCTCA \\
SOD2 & TTGGCCAAGGGAGATGTTAC & AGTCACGTTTGATGGCTTCC \\
\hline
\end{tabular}

GAPDH, glyceraldehyde 3-phosphate dehydrogenase; SOD, superoxide dismutase

it is well established that the oxytocin (OT)/oxytocin receptor (OTR) system plays a central role in the biochemistry of term/preterm labor. Functional OTR is expressed both in the myometrium and fetal membranes (amnion and chorion). OT is widely used for induction/augmentation of both preterm and term labor, and OT binding to its receptor leads to OTR coupling with both $\mathrm{G}_{\alpha q / 11}$ and $\mathrm{G}_{\alpha \mathrm{i} / \mathrm{o}} \mathrm{G}$ proteins (Phaneuf et al., 1993). $G_{\alpha q / 11}$ signaling in the myometrium activates phospholipase $\mathrm{C}$ to drive increases in intracellular $\mathrm{Ca}^{2+}$ via inositol triphosphate, ultimately leading to contractions. Previous studies showed that OTR signaling through $\mathrm{G}_{\alpha i / \mathrm{o}}$ reduces cAMP by inhibition of adenylate cyclase activity (Blanks and Thornton, 2003; Busnelli et al., 2012). We recently demonstrated a novel mechanism for OT driving proinflammatory effects in human gestational tissues (Kim et al., 2015). In the amnion, this was found to be via $G_{\alpha i}$ signaling, which leads to activation of mitogen-activated protein kinases (MAPKs) and nuclear factor- $\kappa \mathrm{B}(\mathrm{NF}-\kappa \mathrm{B})$, and a subsequent increase in the expression of NF- $\kappa \mathrm{B}$-regulated genes including prostaglandin (PG) synthetic enzymes and inflammatory chemokines/cytokines.

OTR antagonists are used in clinical practice for the treatment of preterm labor through inhibition of myometrial contractility, but their effect on the proinflammatory effects of OT has been largely ignored. We recently showed that these OT-driven proinflammatory effects in the amnion are not inhibited by atosiban. Conversely, atosiban alone results in activation of MAPKs and NF- $\kappa$ B to the same extent as OT (Kim et al., 2016). Atosiban acts as a biased ligand to the $G_{\alpha i}$ pathway, which may limit its use to 48 hours (Busnelli et al., 2012; Haas et al., 2012), yet OTR still represents an attractive drug target for tocolysis. Indeed, with growing recognition of the complexity of G protein-coupled receptor (GPCR) signaling, there has been an impetus to design more specific compounds modulating GPCR action that show bias toward specific signaling pathways (Whalen et al., 2011; Busnelli et al., 2012).

Clinical studies to date have used OTR antagonists for acute tocolysis rather than for the prevention of preterm labor in women at high risk for preterm delivery. The only OTR antagonist currently licensed in Europe is atosiban, which can only be given as a continuous intravenous infusion for no more than 48 hours and thus precludes long-term administration for maintenance therapy. Nolasiban is a novel, smallmolecule, nonpeptide OTR antagonist. Nolasiban is selective for OTR against the arginine vasopressin (AVP) V1a and V2 receptors, whereas atosiban is primarily an AVP V1a antagonist (Manning et al., 2001). Whereas the currently available OTR antagonist atosiban is given via intravenous infusion, nolasiban is administered orally and thus lends itself to preterm labor treatment beyond 48 hours.
In this study, we investigated whether the OTR antagonist nolasiban can inhibit OT-driven activation of the inflammatory response in human gestational tissues, and we compared and contrasted the effects of nolasiban with the currently licensed OTR antagonist atosiban.

\section{Materials and Methods}

Cell Culture. Placentas with fetal membranes and myometrial biopsies were collected from term (38-40 weeks of pregnancy), nonlaboring women with singleton pregnancies undergoing scheduled low-risk elective caesarean section. This study excluded individuals who had preexisting medical conditions, preeclampsia, and/or multiple pregnancies or had received uterotonics prior to their caesarean sections. Samples were collected after participants gave informed consent and the study was approved by the local research ethics committees of Queen Charlotte's and Chelsea Hospital (placenta, RREC 2002-6283; and myometrium, RREC 1997-5089).

The amnion layer from the placenta was pulled apart from the chorion to use for culture. Primary amnion epithelial cells were established from fetal membranes, as previously described (Bennett et al., 1987). For myometrial smooth muscle cell isolation, the biopsies were dissected into fine pieces and digested in filter-sterilized collagenase $\mathrm{mix}$ [ $1 \mathrm{mg} / \mathrm{ml}$ collagenase $1 \mathrm{~A}, 1 \mathrm{mg} / \mathrm{ml}$ collagenase $\mathrm{X}$, and $2 \mathrm{mg} / \mathrm{ml}$ bovine serum albumin in 1:1 ratio of Dulbecco's modified Eagle's medium (DMEM)/Ham's F-12 Nutrient Mixture and serumfree DMEM; Sigma-Aldrich, St. Louis, MO] for 45 minutes at $37^{\circ} \mathrm{C}$. The digestion process was halted using DMEM with $10 \%$ (v/v) fetal calf serum and the cell suspension was obtained by filtering out the tissue through a $40-\mu \mathrm{m}$ cell strainer (Corning, Corning, NY). Centrifugation of the suspension at $3000 \mathrm{rpm}$ for 5 minutes collected the cells into a pellet, which was subsequently resuspended in full DMEM (10\% fetal calf serum, $2 \mathrm{mM} \mathrm{L}$-Glutamine, and $100 \mathrm{U} / \mathrm{ml}$ penicillin-streptomycin) and cultured in cell culture-treated flasks (Corning) at $37^{\circ} \mathrm{C}$ in

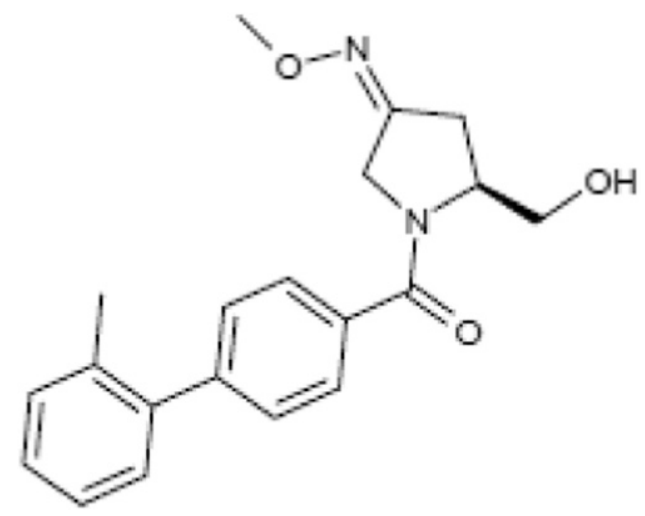

Fig. 1. Chemical structure of nolasiban. 


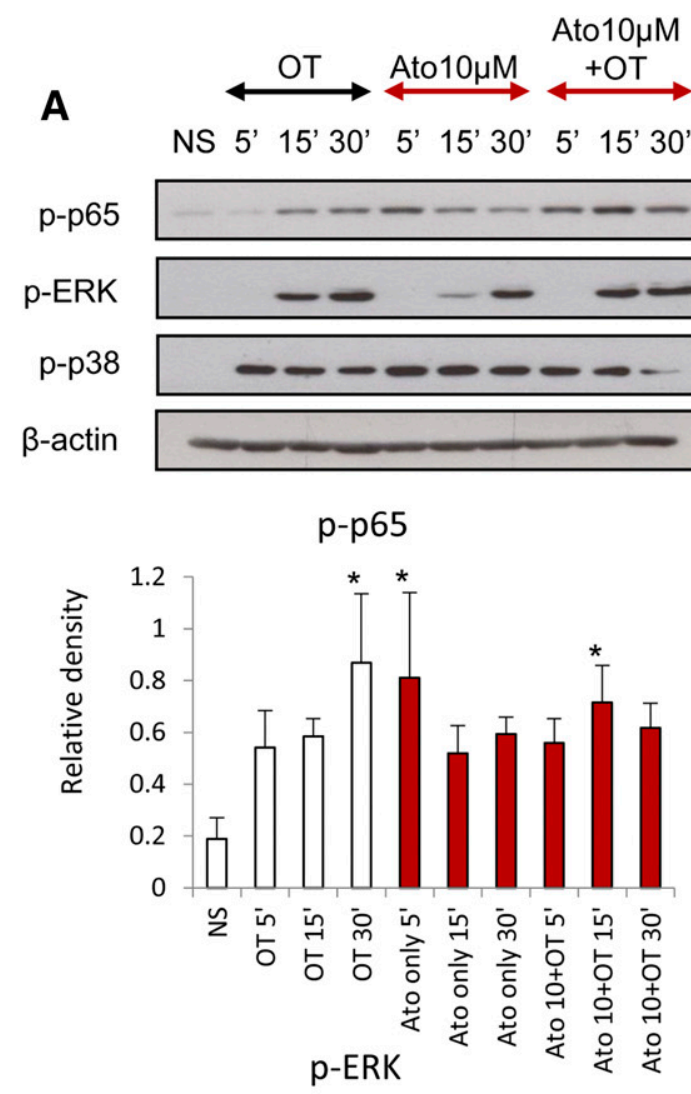

B

Ato $10 \mu \mathrm{M}$

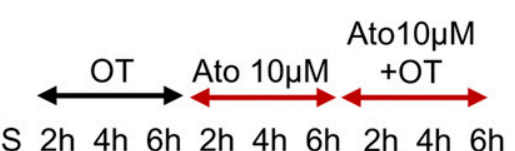

NS $2 \mathrm{~h} 4 \mathrm{~h} 6 \mathrm{~h} 2 \mathrm{~h} 4 \mathrm{~h} 6 \mathrm{~h} 2 \mathrm{~h} 4 \mathrm{~h} 6 \mathrm{~h}$
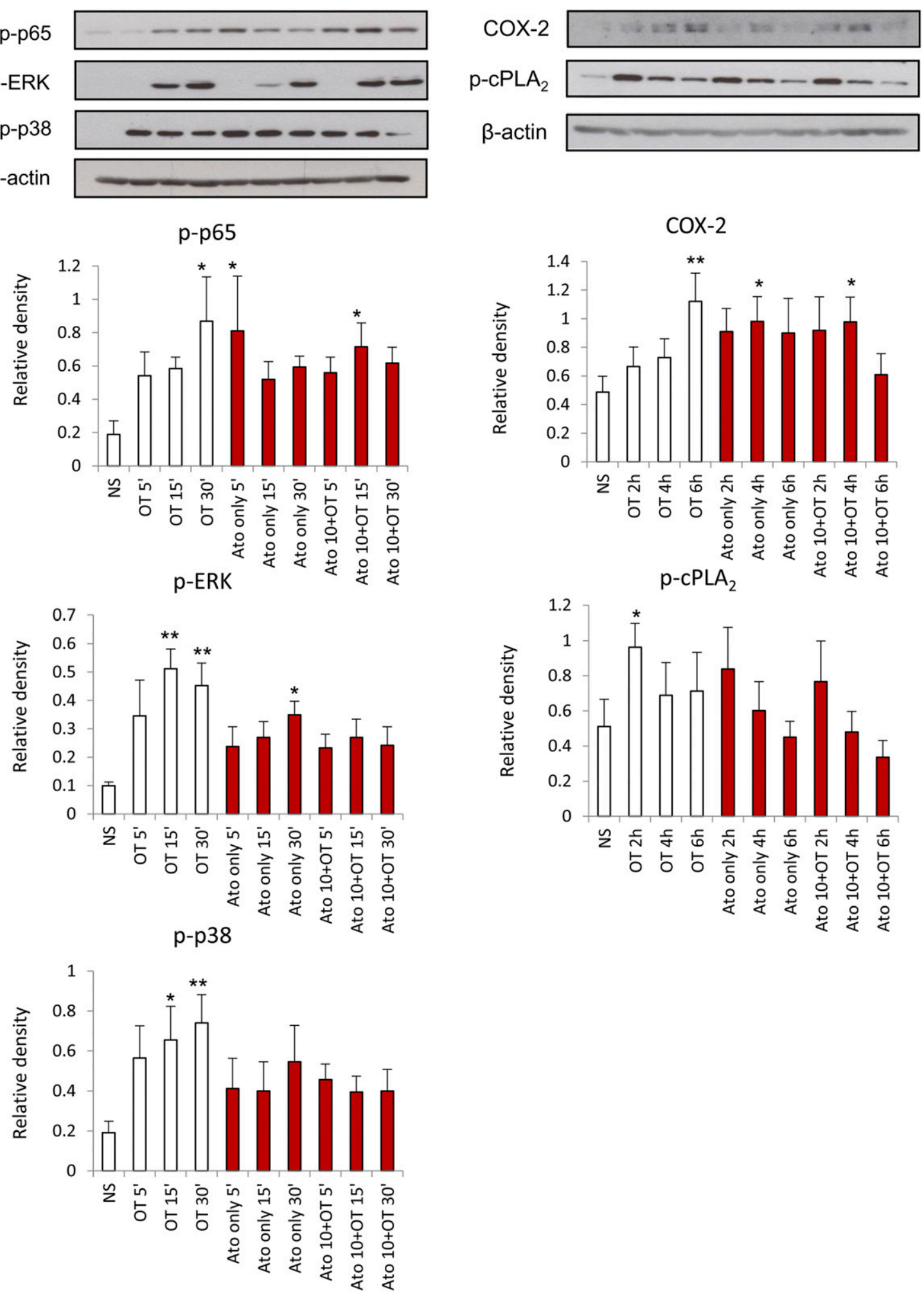

Fig. 2. The effect of atosiban on OT-induced proinflammatory responses in amnion cells. Prelabor amnion epithelial cells were stimulated for 5 minutes, 15 minutes, 30 minutes, 2 hours, 4 hours, and 6 hours with atosiban $(10 \mu \mathrm{M})$ and/or OT $(10 \mathrm{nM})$. (A and B) Representative Western blots for the phosphorylated NF- $\kappa$ B p65 subunit, ERK1/2, and p38 MAPK (A), as well as COX-2 and p-cPLA 2 (B), are shown above their corresponding densitometry graphs with fold changes compared with NS controls. Matched $\beta$-actin loading controls confirm equal loading $(n=6$; $* P<0.05$; ** $P<0.01$ compared with NS). Significance was tested using ANOVA, with correction for multiple testing by the Tukey-Kramer multiple comparisons test. ANOVA, analysis of variance; Ato, atosiban; NS, nonstimulated. 

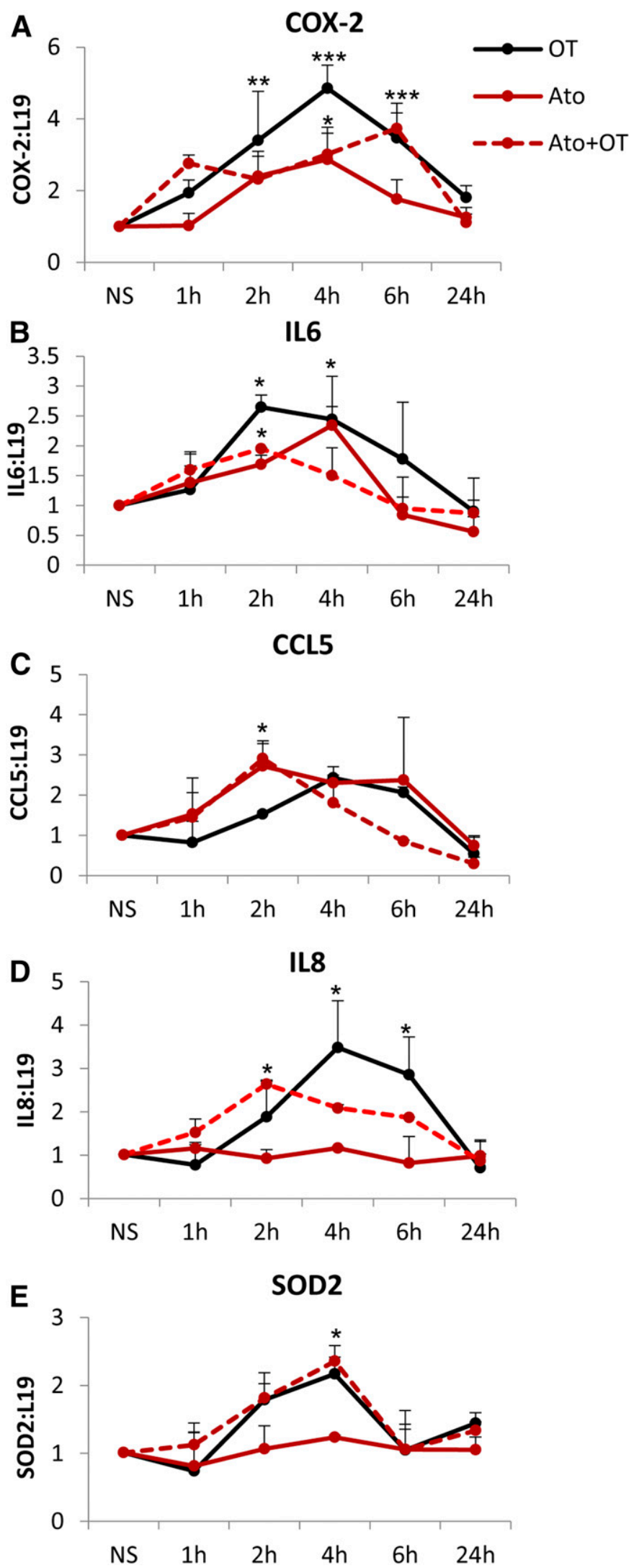

Fig. 3. Increase in prolabor NF- $\kappa \mathrm{B}-$ regulated gene expression with atosiban stimulation in amnion cells. Prelabor amnion epithelial cells were stimulated for 1 hour, 2 hours, 4 hours, and 6 hours with atosiban $(10 \mu \mathrm{M})$ and/or OT $(10 \mathrm{nM})$. (A-E) The expression of downstream NF- $\kappa \mathrm{B}-$ regulated genes, COX-2 (A), IL-6 (B), CCL5 (C), IL-8 (D), and SOD2 (E), was examined from total RNA extracts. Atosiban had no effect on humidified atmosphere of $95 \%$ air and $5 \% \mathrm{CO}_{2}$. When myometrial cells reached $95 \%$ confluence, they were passaged using $0.25 \%$ trypsin with $0.02 \%$ EDTA in phosphate-buffered saline. We used myometrial cells between passage numbers one to four.

Real-Time Polymerase Chain Reaction. Extraction of total RNA was carried out using RNA STAT-60 reagent for guanidinium thiocyanate-phenol-chloroform extraction (AMS Biotechnology, Abingdon, UK), following the manufacturer's recommended protocol. Any potential DNA contaminations were eliminated from the extracted RNA by DNase I treatment (Invitrogen, Carlsbad, CA) prior to cDNA synthesis. The DNase I-treated RNA was used for first-strand cDNA synthesis by Moloney murine leukemia virus reverse transcriptase (Sigma-Aldrich). Changes in gene expression were determined by realtime polymerase chain reaction with the ABI StepOne Plus Real-Time PCR system (Applied Biosystems, Foster City, CA) using SYBR Green JumpStart Taq ReadyMix (Sigma-Aldrich). Primer Express software (Applied Biosystems) was used to design specific primers for each gene. Sequences of the gene-specific primers are listed in Table 1. The resulting data were analyzed using StepOne software (version 2.3; Applied Biosystems). All primers were tested for efficiency, and the comparative cycle threshold method was used to analyze the relative changes in gene expression. Cycle threshold values for all target genes were normalized to constitutively expressed ribosomal protein L19 and also to glyceraldehyde 3-phosphate dehydrogenase for amnion and myometrial cells, respectively.

Western Blot Analysis. Whole-cell lysates were obtained by lysing the monolayer of cells in modified radioimmunoprecipitation assay buffer, containing $150 \mathrm{mM} \mathrm{NaCl}, 10 \mathrm{mM}$ Tris (pH 7.4), $1 \%$ Triton $\mathrm{X}-100,0.1 \%$ SDS, $1 \%$ sodium deoxycholate, $1 \mathrm{mM}$ EDTA, $1 \mathrm{mM}$ phenylmethylsulfonyl fluoride, and protease and phosphatase inhibitor cocktails (Sigma-Aldrich; Thermo Fisher, Rockford, IL). The concentration of each lysate was determined using a detergent compatible protein assay kit (Bio-Rad, Hercules, CA) and $40 \mu \mathrm{g}$ of each protein sample was denatured in loading dye with $50 \mathrm{mM}$ dithiothreitol by heating for 10 minutes at $80^{\circ} \mathrm{C}$. Proteins were separated by electrophoresis on a $10 \%$ SDS-polyacrylamide gel for 80 minutes at constant $140 \mathrm{~V}$ and were transferred to a polyvinylidene difluoride membrane (Millipore, Billerica, MA) in a wet-transfer chamber system (Bio-Rad) for 90 minutes at constant $300 \mathrm{~mA}$. A precast midi-gel (Bio-Rad) was used to separate more than 15 protein samples. Polyvinylidene difluoride membranes were blocked in blocking buffer [5\% (w/v) milk powder in Tris-buffered saline with $0.1 \%$ Tween-20] prior to primary antibody incubation overnight at $4^{\circ} \mathrm{C}$. Horseradish peroxidase-conjugated secondary antibody incubation lasted for 1 to 2 hours at room temperature, and signal detection was carried out using an enhanced chemiluminescence reagent (Bio-Rad). To confirm equal loading of samples, membranes were treated with a mild stripping buffer $(0.2 \mathrm{M} \mathrm{NaOH})$ and reprobed for $\beta$-actin.

Enzyme-Linked Immunosorbent Assay. Concentrations of $\mathrm{PGE}_{2}$ in the culture supernatants were determined by a standard enzyme-linked immunosorbent assay. At treatment completion, culture media from both myometrial and amnion cells were collected and immediately frozen for storage at $-20^{\circ} \mathrm{C}$ until subsequent analysis by enzyme-linked immunosorbent assay, following the manufacturer's instructions (R\&D Systems, Minneapolis, MN).

Materials. Goat anti-cyclooxygenase (COX)-2 (C20) antibody (Santa Cruz Biotechnology, Dallas, TX) was used at 1:2000 dilution, and rabbit monoclonal antibodies to phosphorylated cytosolic phospholipase $\mathrm{A}_{2}$ (p-cPLA ${ }_{2}$ ), p-p65 (Ser536), p-MAPK14 (p38 MAPK), and p-MAPK3/1 (extracellular signal-regulated kinase ERK1/2 p44/42

OT-induced upregulation, but atosiban alone increased the expression of COX-2, IL-6, and CCL5 $(n=6 ; * P<0.05 ; * * P<0.01 ; * * * P<0.001$ compared with NS; ANOVA). Significance was tested using ANOVA, with correction for multiple testing by the Tukey-Kramer multiple comparisons test. ANOVA, analysis of variance; Ato, atosiban; NS, nonstimulated; SOD, superoxide dismutase. 
A

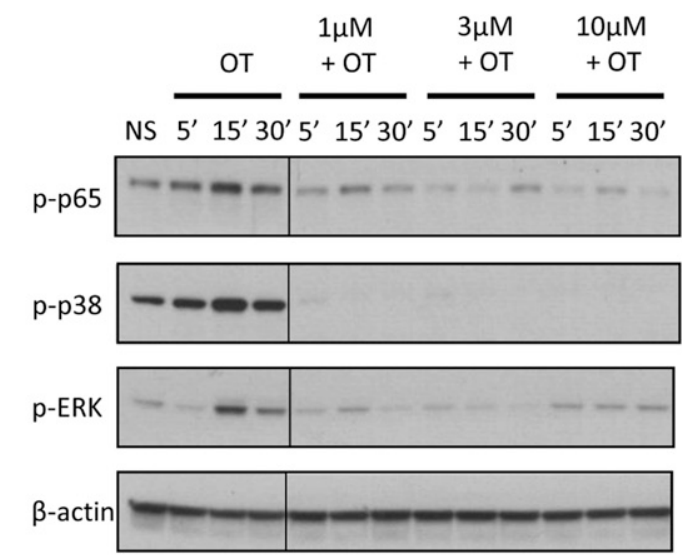

B
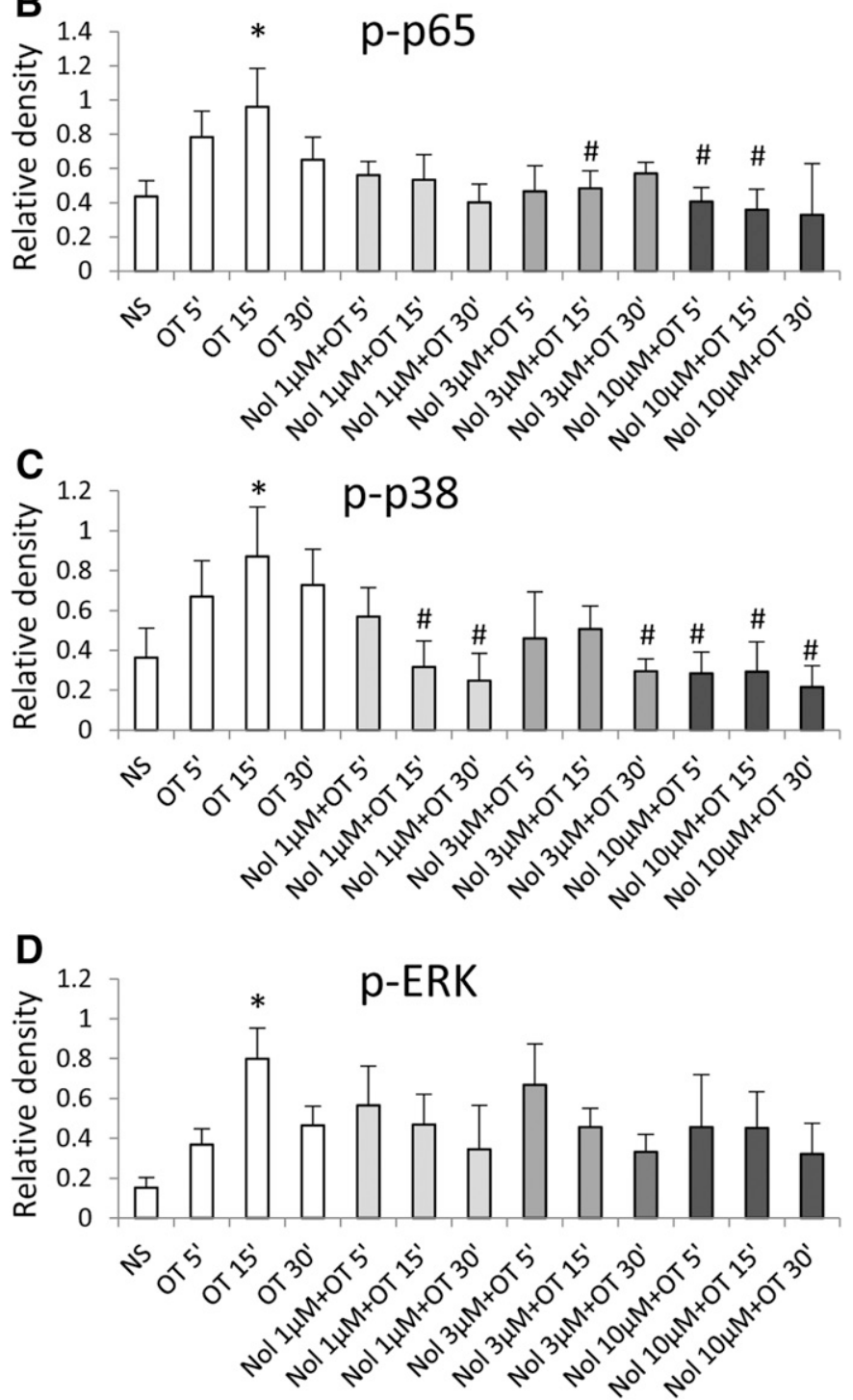

Fig. 4. The effect of nolasiban on OT-mediated activation of NF- $\kappa \mathrm{B}$ and MAPKs in amnion cells. Prelabor primary amnion epithelial cells were stimulated with OT $(10 \mathrm{nM})$ in the presence or absence of nolasiban $(1,3$, or $10 \mu \mathrm{M}$ ) for 5 minutes, 15 minutes, and 30 minutes. (A) Western blots were used to examine the effect of nolasiban on OT-mediated activation of p65 $\mathrm{NF}-\kappa \mathrm{B}$ and MAPKs, ERK1/2, and p38 MAPK. Solid lines indicated cropping boundaries of strips (please note that this editing is also done in the blot panels in Figs. 5A, 10A, and 11A). NS controls and OT-only treatments were adjusted to contain the vehicle, DMSO, at $0.1 \%(\mathrm{v} / \mathrm{v})$.
MAPK) from Cell Signaling Technology (Danvers, MA) were used at $1: 1000$ dilution. The mouse monoclonal anti- $\beta$-actin antibody (at 1:50,000) was from Abcam (Cambridge, UK). Horseradish peroxidaseconjugated secondary antibodies against goat, mouse, and rabbit IgGs were from Santa Cruz Biotechnology. Oxytocin and atosiban were obtained from Alliance Pharmaceuticals (San Diego, CA) and SigmaAldrich, respectively. Nolasiban was synthesized as previously described (Chollet, 2015). The nolasiban chemical formula is $(3 Z, 5 S)$ 5-(hydroxymethyl)-1-[(2'-methyl-1,1'-biphenyl-4-yl)carbonyl]pyrrolidin3 -one $O$-methyloxime (Fig. 1). Nolasiban is prepared following an eight-step synthesis starting from 4-bromobenzoic acid. A detailed description of nolasiban preparation is provided below.

A solution of potassium carbonate in water was charged to a slurry of 4 -bromobenzoic acid in water at $15-25^{\circ} \mathrm{C}$. The resulting slurry was stirred at $15-25^{\circ} \mathrm{C}$ and degassed three times using a vacuum-nitrogen purge cycle. Tetrakis(triphenylphosphine)palladium(0) was charged and the vacuum-nitrogen purge cycle was repeated. A solution of $o$-tolylboronic acid was degassed three times, using a vacuum-nitrogen purge cycle, and then charged to the 4-bromobenzoic acid slurry at 15$25^{\circ} \mathrm{C}$. The reaction mixture was heated to and maintained at reflux until reaction completion (the reaction is considered complete at $95 \%$ conversion), as determined by proton nuclear magnetic resonance spectroscopy ( ${ }^{1} \mathrm{H}$ NMR) analysis [ $d_{6}$-dimethylsulfoxide (DMSO)], typically $1.5-2.5$ hours. After workup, the solid was dried in a vacuum oven at $35-40^{\circ} \mathrm{C}$ to give 4 -(2-methylphenyl)benzoic acid $\left[{ }^{1} \mathrm{H}\right.$ NMR $\left(d_{6^{-}}\right.$ DMSO) concordant with structure] as a pale yellow solid. Thionyl chloride was added to a slurry of 4-(2-methylphenyl)benzoic acid in toluene at $10-25^{\circ} \mathrm{C}$ and the mixture was heated to and maintained at $75-80^{\circ} \mathrm{C}$ until complete by ${ }^{1} \mathrm{H}$ NMR analysis ( $d_{6}$-benzene), typically 4 to 5 hours. After workup, the toluene solution of 4-(2-methylphenyl)benzoic acid chloride $\left[{ }^{1} \mathrm{H}\right.$ NMR $\left(d_{6}\right.$-benzene) concordant with structure] was used directly in the next step.

A solution of potassium carbonate was charged to a solution of 4-hydroxy-L-proline in tetrahydrofuran and water at $15-25^{\circ} \mathrm{C}$ followed by a line rinse of water. The mixture was cooled to $0-5^{\circ} \mathrm{C}$ with rapid stirring and a solution of 4-(2-methylphenyl)benzoic acid chloride in toluene was charged at that temperature followed by a line rinse of toluene. The reaction mixture was warmed to $15-25^{\circ} \mathrm{C}$ over 1 to 2 hours and stirred at this temperature until judged complete by thin layer chromatography (TLC) analysis. After workup, the collected solid was dried under vacuum at $40-45^{\circ} \mathrm{C}$ until the water content by Karl Fischer titration was $\leq 0.2 \%$ (w/w) to afford (4R)-4-hydroxy-1[(2'-methyl-1,1'-biphenyl-4-yl)carbonyl]-L-proline [ ${ }^{1} \mathrm{H}$ NMR $\left(d_{6}\right.$-DMSO) concordant with structure] as an off-white solid.

Triethylamine was charged to a solution of $(4 R)-4$-hydroxy-1-[(2'methyl-1,1'-biphenyl-4-yl)carbonyl]-L-proline in DMSO at $15-20^{\circ} \mathrm{C}$. Pyridine-sulfur trioxide complex was charged portion-wise at 15 and $25^{\circ} \mathrm{C}$ and the reaction mixture was stirred at that temperature until reaction completion, as determined by TLC analysis (typically 1-3 hours). After workup, the collected solid was dried under vacuum at 40$45^{\circ} \mathrm{C}$ to give 1-[(2'-methyl-1,1'-biphenyl-4-yl)carbonyl]-4-oxo-L-proline [ ${ }^{1} \mathrm{H}$ NMR $\left(d_{6}\right.$-DMSO) concordant with structure] as an off-white solid.

Triethylamine was added to a solution of 1-[(2'-methyl-1,1'-biphenyl4-yl)carbonyl]-4-oxo-L-proline in dichloromethane at $10-25^{\circ} \mathrm{C}$. Methoxylamine hydrochloride was added portion-wise at $10-25^{\circ} \mathrm{C}$ and the reaction mixture was stirred at $10-25^{\circ} \mathrm{C}$ until reaction completion, as determined by TLC analysis [typically $3-5$ hours, TLC eluent: dichloromethane/methanol/acetic acid (90:10:1); UV visualization]. After workup, the filtrate and washes were combined and concentrated

$\beta$-actin confirmed equal protein loading. (B-D) Densitometric analyses showed significant decreases in OT-mediated activation of p65 (B) and p38 (C) in the presence of nolasiban, but not ERK1/2 (D) $(n=6$; $* P<0.05$ versus NS; ${ }^{\#} P<0.05$ versus OT-treated samples; ANOVA). Significance was tested using ANOVA, with correction for multiple testing by TukeyKramer multiple comparisons test. ANOVA, analysis of variance; Nol, nolasiban; NS, nonstimulated. 
A

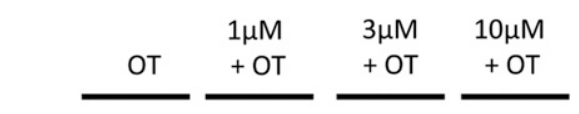

NS $2 \mathrm{~h} 4 \mathrm{~h} 6 \mathrm{~h} 2 \mathrm{~h} 4 \mathrm{~h} 6 \mathrm{~h} 2 \mathrm{~h} 4 \mathrm{~h} 6 \mathrm{~h} 2 \mathrm{~h} 4 \mathrm{~h} 6 \mathrm{~h}$
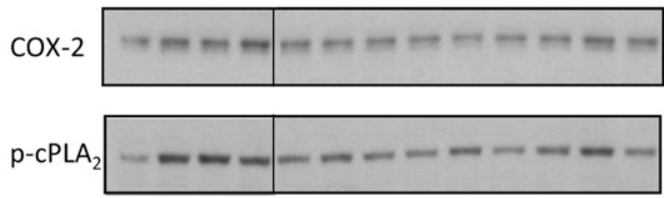

$\beta$-actin
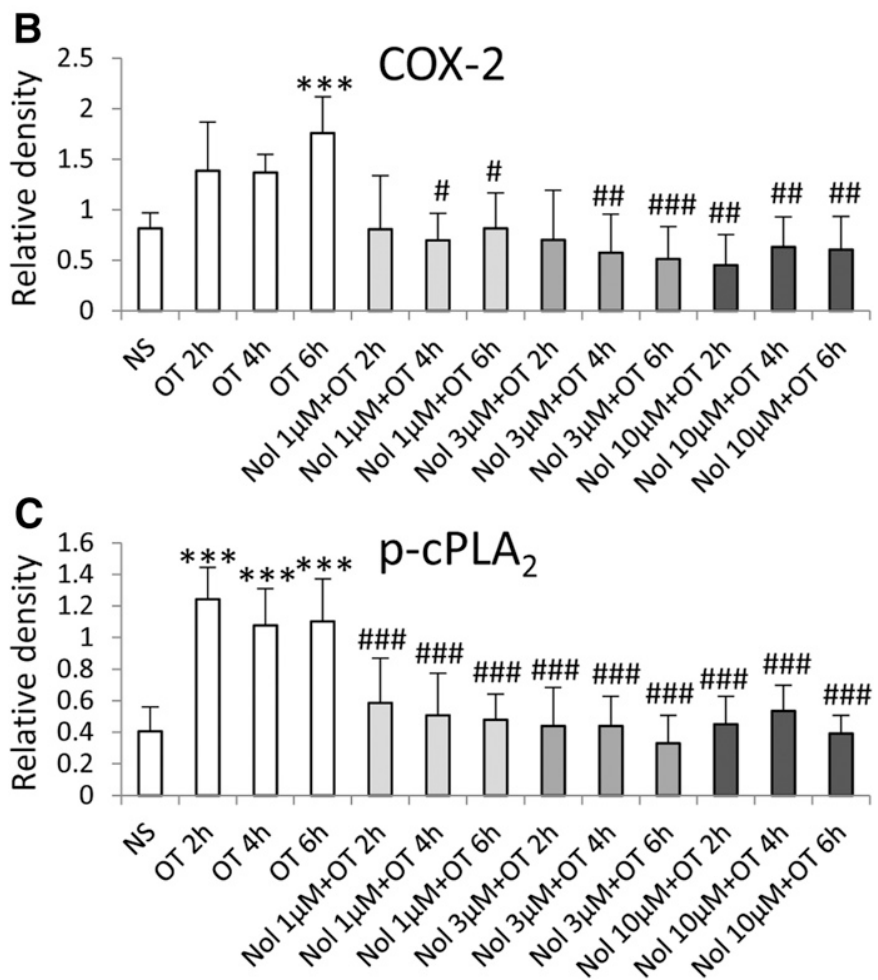

Fig. 5. The effect of nolasiban on OT-mediated expression of PG synthetic enzymes in amnion cells. Prelabor primary amnion epithelial cells were stimulated with OT $(10 \mathrm{nM})$ in the presence or absence of nolasiban $(1,3$, or $10 \mu \mathrm{M}$ ) for 2 hours, 4 hours, and 6 hours. (A) Western blots were used to examine the effect of nolasiban on OT-mediated expression of COX-2 and p-cPLA ${ }_{2}$. NS controls and OT-only treatments were adjusted to contain the vehicle, DMSO, at $0.1 \%$ (v/v). $\beta$-actin confirmed equal protein loading. (B and C) Densitometric analyses showed significant decreases in OT-mediated expression of COX-2 (B) and p-cPLA $2(\mathrm{C})$ in the presence of nolasiban $(n=6$; ${ }^{* * *} P<0.001$ versus NS; ${ }^{\#} P<0.05$; ${ }^{\# \#} P<0.01 ;{ }^{\# \# \#} P<0.001$ versus OT-treated samples; ANOVA). Significance was tested using ANOVA, with correction for multiple testing by the Tukey-Kramer multiple comparisons test. ANOVA, analysis of variance; Nol, nolasiban; NS, nonstimulated.

under vacuum at $35-40^{\circ} \mathrm{C}$ to afford 4-methoxyimino-1-[(2'-methyl-1,1'biphenyl-4-yl)carbonyl]-L-proline $\left[{ }^{1} \mathrm{H}\right.$ NMR (CDCl3) concordant with structure] as an off-white solid.

Potassium carbonate was added to a solution of 4-methoxyimino-1-[ $(2$ 'methyl-1,1'-biphenyl-4-yl)carbonyl]-L-proline and the mixture was cooled to $0-10^{\circ} \mathrm{C}$. Dimethyl sulfate was added at $0-15^{\circ} \mathrm{C}$ and the mixture was stirred at $15-25^{\circ} \mathrm{C}$ until reaction completion, as determined by TLC analysis, typically 3-16 hours. After workup, excess ethyl acetate was removed by azeotropic distillation with tetrahydrofuran under vacuum at $40-45^{\circ} \mathrm{C}$ to give $(4 Z / E, 2 S)$-methyl-1-[(2' -methyl-1,1'-biphenyl-4-yl)carbonyl]-4-methoxyimino pyrrolidine-2-carboxylate [ ${ }^{1} \mathrm{H}$ NMR (CDCl3) concordant with structure] as a viscous brown oil.
Lithium borohydride was added portion-wise under nitrogen to a stirred solution of $(4 Z / E, 2 S)$-methyl-1-[(2'-methyl-1,1'-biphenyl-4yl)-carbonyl]-4-methoxyimino pyrrolidine-2-carboxylate in tetrahydrofuran and methanol at $0-30^{\circ} \mathrm{C}$. The prepared mixture was stirred at $15-25^{\circ} \mathrm{C}$ to reaction completion, as determined by TLC analysis (eluent: ethyl acetate; visualization: ninhydrin), typically 2-6 hours. After workup, the combined filtrate and wash were concentrated under vacuum to give a crude isomeric mixture comprising (3Z,5S)-5-(hydroxymethyl)-1-[(2'-methyl-1,1'-biphenyl-4-yl)carbonyl]pyrrolidin-3-one $O$-methyloxime and $(3 E, 5 S)-5$-(hydroxymethyl)-1-[(2'-methyl-1,1'biphenyl-4-yl)carbonyl]pyrrolidin-3-one $O$-methyloxime $\left[{ }^{1} \mathrm{H}\right.$ NMR (CDCl3) concordant with structure; $82.0 \%$ area by high-pressure liquid chromatography, 71.4:28.6 $\mathrm{Z} / \mathrm{E}$ ratio] as a viscous brown oil. The oil was dissolved in toluene and stored until required. Pure nolasiban or $(3 Z, 5 S)$-5-(hydroxymethyl)-1-[(2'-methyl-1,1'-biphenyl4-yl)carbonyl]pyrrolidin-3-one $O$-methyloxime was isolated by chromatography and elution with toluene/MeOH [99:1 (v/v)].

The vehicle controls for OT, atosiban and nolasiban were DMEM (Sigma-Aldrich), double deionized water, and DMSO, respectively. Nolasiban was diluted from stock solutions using DMEM shortly prior to the treatments. The DMSO concentration was adjusted to $0.1 \%(\mathrm{v} / \mathrm{v})$ in all dose formulations and control wells for nolasiban experiments were treated to contain $0.1 \%(\mathrm{v} / \mathrm{v})$ DMSO.

Statistical Analysis. GraphPad Prism software (version 5.0; GraphPad Inc., La Jolla, CA) was used for statistical analysis. We tested our data sets for normality using the Kolmogorov-Smirnov test prior to appropriate statistical comparisons. When data were normally distributed, multiple comparisons were performed using analysis of variance, followed by the Tukey-Kramer (or the Tukey honest significant difference) post hoc test. For non-normally distributed data, multiple comparisons were performed using the Freidman test, followed by the Dunn multiple comparisons post hoc test. In this study, all data sets are presented as means \pm S.E.M. and differences were considered statistically significant at $P<$ 0.05 .

\section{Results}

Atosiban Has No Effect on OT-Mediated Upregulation of Proinflammatory Mediators But Mimics the Effects of OT in Human Amnion. We previously demonstrated that the presence of atosiban in amnion cells has no significant effect on the OT-driven activation of NF- $\kappa \mathrm{B}$ and MAPKs and the upregulation of downstream prolabor genes at an OT concentration of $100 \mathrm{nM}$ (Kim et al., 2016). In this study, amnion cells were treated with atosiban and a lower dose of OT (10 nM), which is more physiologically relevant. There was no significant inhibition of OT effects in the presence of atosiban; similar to recently published data, atosiban consistently led to activation of proinflammatory mediators including NF- $\kappa \mathrm{B}$ and MAPKs and subsequent expression of PG synthetic enzymes COX-2 and p-cPLA 2 (Fig. 2).

These effects of atosiban translated to changes in the expression of downstream NF- $\kappa \mathrm{B}-$ regulated genes. The presence of atosiban did not inhibit OT-mediated expression of COX-2, interleukin (IL)-6, C-C motif chemokine ligand CCL5, IL-8, and superoxide dismutase 2 at the mRNA level (Fig. 3). Furthermore, atosiban treatment alone resulted in 2.8-, 2.3-, and 2.4-fold increases in the expression of COX-2, IL-6, and CCL5 (all $P<0.05$ ), respectively (Fig. 3, A-C).

Unlike Atosiban, Nolasiban Suppresses the Effects of OT on Proinflammatory Mediators Including Activation of NF- $\kappa$ B and MAPKs and Prolabor Gene Expression in Human Amnion. Matching experiments in amnion cells 


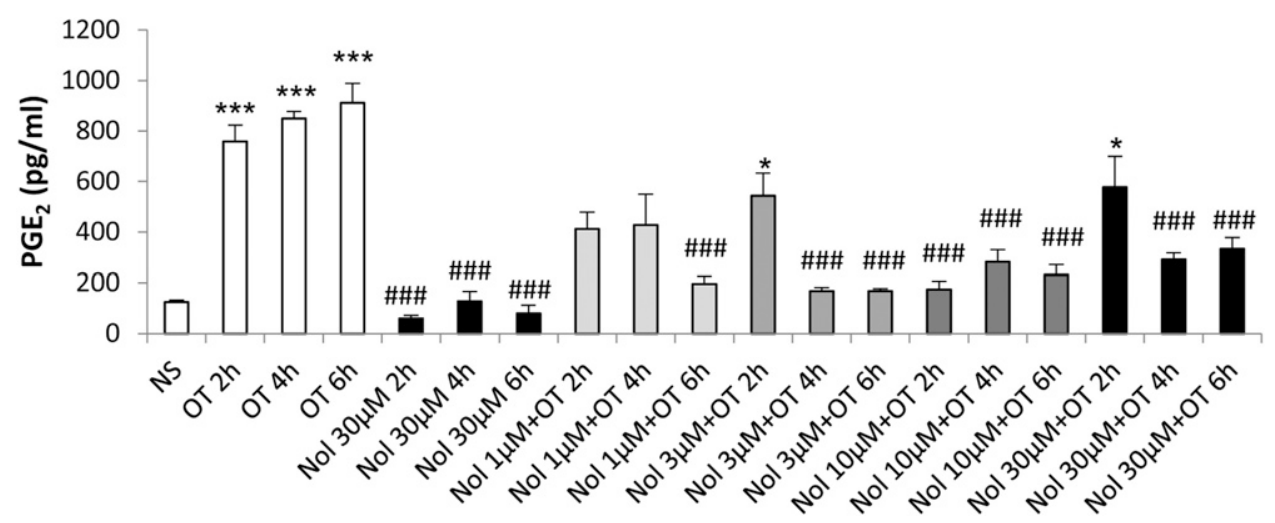

Fig. 6. The effect of nolasiban on OTmediated $\mathrm{PGE}_{2}$ release in amnion cells. Prelabor primary amnion epithelial cells were stimulated with OT (10 $\mathrm{nM})$ in the presence or absence of nolasiban $(1,3,10$, or $30 \mu \mathrm{M}$ ) for 2 hours, 4 hours, and 6 hours. The $\mathrm{PGE}_{2}$ enzyme-linked immunosorbent assay demonstrated a significant inhibition of OT-mediated $\mathrm{PGE}_{2}$ production by nolasiban $(n=6$; $* P<0.05 ; * * * P<0.001$ versus NS; ${ }^{\# \#} P<0.001$ versus OT-treated samples; ANOVA). Significance was tested using ANOVA, with correction for multiple testing by the Dunn multiple comparisons test. ANOVA, analysis of variance; Nol, nolasiban; NS, nonstimulated. using nolasiban showed markedly different results to atosiban. The presence of nolasiban led to a significant reduction in OT-driven activation of both NF- $\kappa \mathrm{B}$ p65 and p38 kinases at an equimolar concentration of atosiban as well as at lower doses (Fig. 4, A-C). There was a slight reduction in OT-induced ERK1/2 activation in a dosedependent manner; however, this did not reach significance (Fig. 4D). Nolasiban alone did not have a significant effect on NF- $\kappa \mathrm{B}$ and p38 activation or expression of PG synthetic enzymes (Supplemental Fig. 1). However, at the maximum dose of nolasiban $(30 \mu \mathrm{M})$, we observed activation of ERK1/2 $(P<0.001)$ with no effect on downstream targets; this indicates that at this high concentration of nolasiban (which is physiologically irrelevant), the effect may be due to activation of an alternative signaling pathway, independent to that of OT.
A

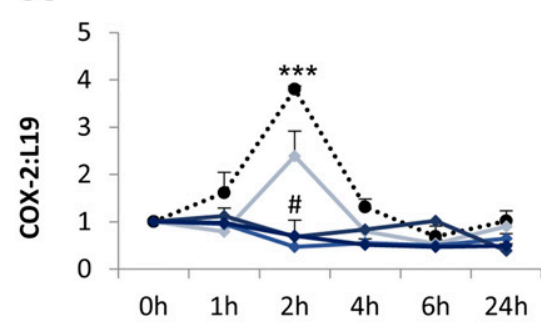

B

IL8

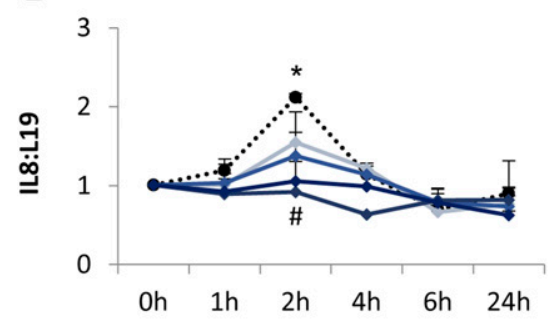

C

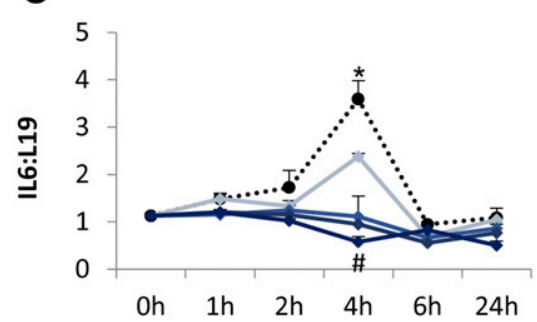

D
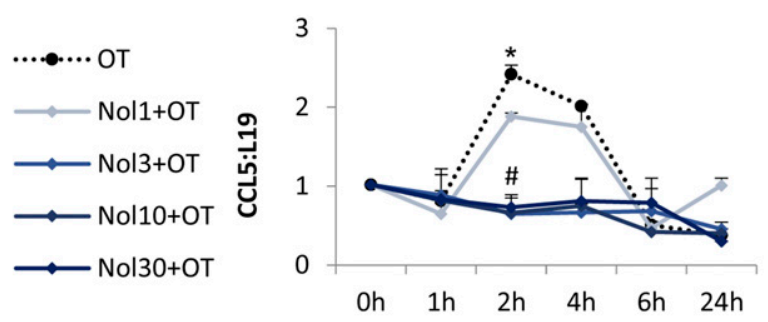

E

CCL2

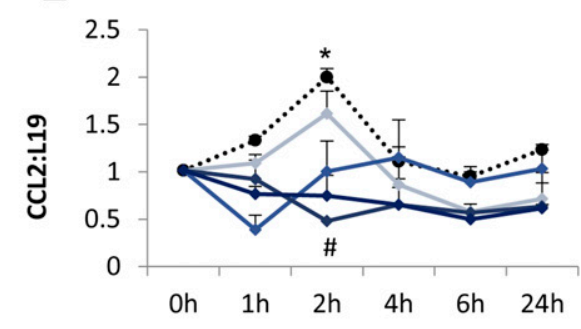

$\mathbf{F}$

SOD2

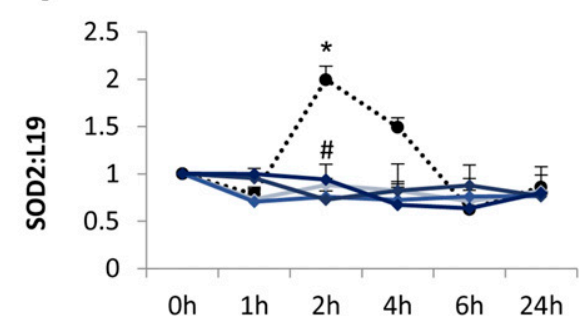

Fig. 7. The effect of nolasiban on OT-mediated expression of downstream NF- $\kappa$ B-regulated genes in amnion cells. (A-F) Prelabor primary amnion epithelial cells were treated with OT $(10 \mathrm{nM})$ in the presence or absence of nolasiban $(1,3,10$, or $30 \mu \mathrm{M})$ for 1 hour, 2 hours, 4 hours, 6 hours, and 24 hours, and changes in the expression of downstream NF- $\mathrm{B}$-regulated genes, COX-2 (A), IL-8 (B), IL-6 (C), CCL5 (D), CCL2 (E), and SOD2 (F), were analyzed using qRT-PCR. For qRT-PCR analysis, target gene mRNA levels were normalized to that of the housekeeping gene, L19 $(n=4 ; * P<0.05$; *** $P<0.001$ versus NS; ${ }^{P} P<0.05$ versus OT-treated samples; ANOVA). Significance was tested using ANOVA, with correction for multiple testing by the TukeyKramer multiple comparisons test. ANOVA, analysis of variance; Nol, nolasiban; qRT-PCR, quantitative real-time polymerase chain reaction; SOD, superoxide dismutase. 

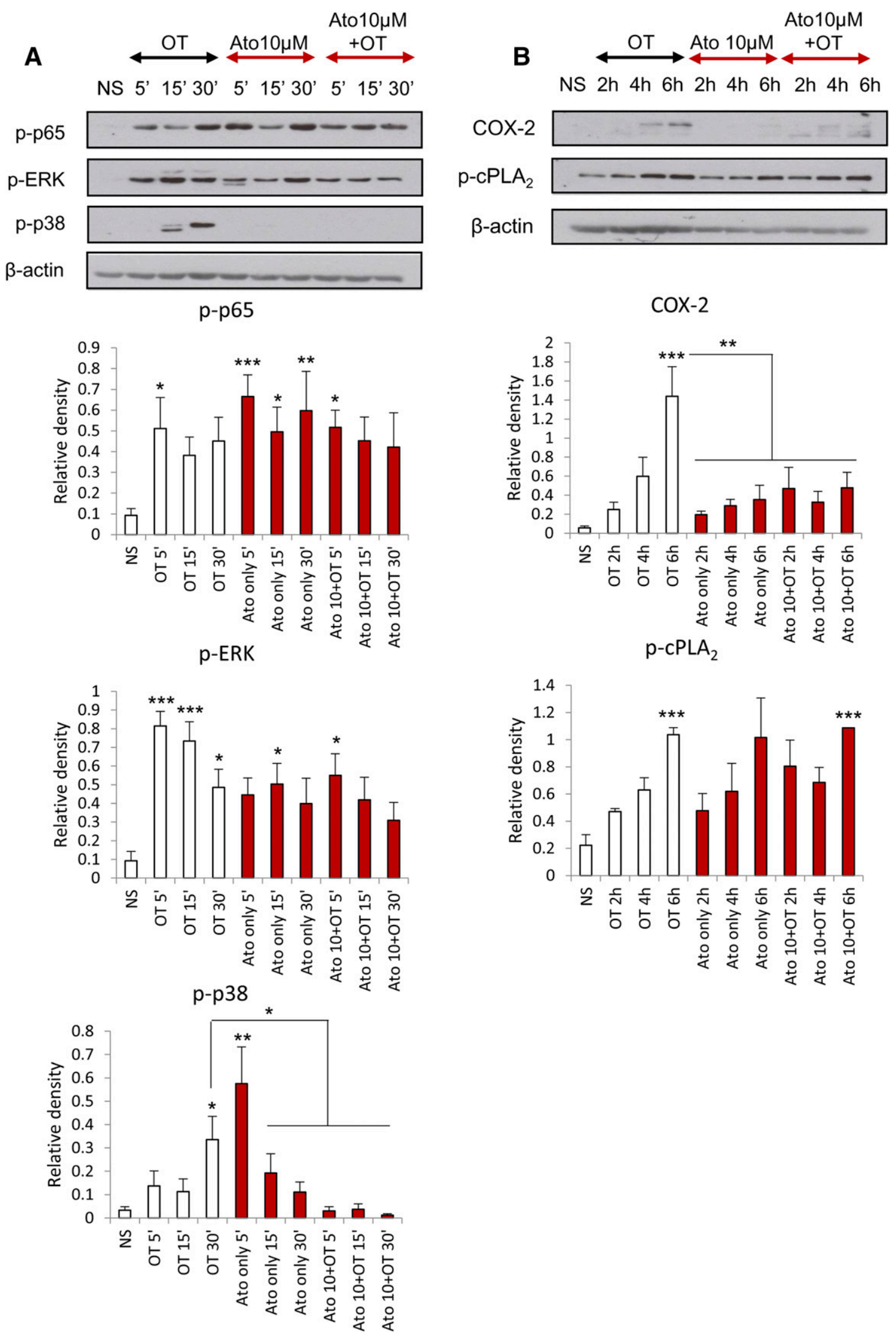

Fig. 8. The effect of atosiban on OT-induced proinflammatory responses in myometrial cells. Prelabor myometrial smooth muscle cells were stimulated with OT $(10 \mathrm{nM})$ for 5 minutes, 15 minutes, 30 minutes, 2 hours, 4 hours, and 6 hours in the presence or absence of atosiban $(10 \mu \mathrm{M})$. (A and $\mathrm{B})$ Representative Western blots for the phosphorylated NF- $\kappa$ B p65 subunit, ERK1/2, and p38 MAPK (A), as well as COX-2 and p-cPLA 2 (B), are shown above their corresponding densitometry graphs with fold changes compared with nonstimulated controls. Matched $\beta$-actin loading controls confirm equal loading $(n=6 ; * P<0.05 ; * * P<0.01 ; * * * P<0.001$ compared with NS; ANOVA). Significance was tested using ANOVA, with correction for multiple testing by the Tukey-Kramer multiple comparisons test. ANOVA, analysis of variance; Ato, atosiban; NS, nonstimulated. 

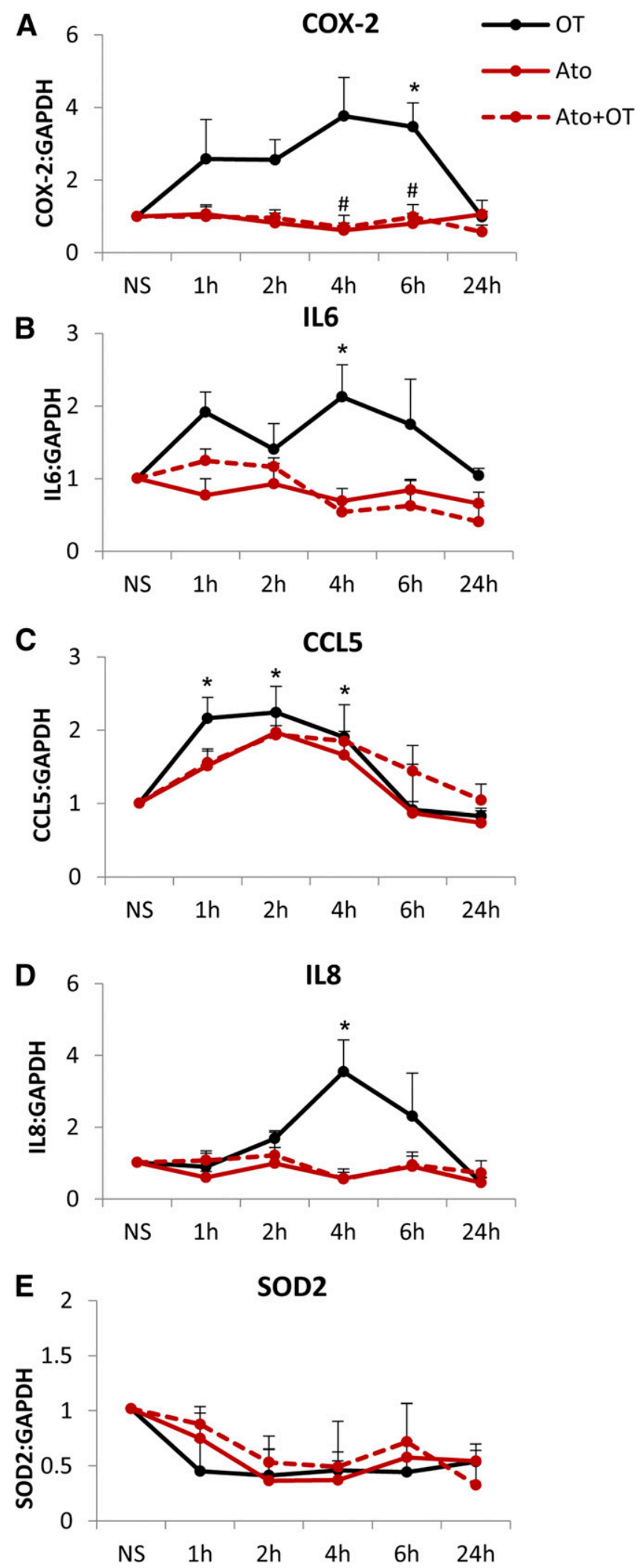

Fig. 9. The effect of atosiban on OT-induced upregulation of NF- $\kappa$ Bregulated genes in myometrial cells. Prelabor myometrial smooth muscle cells were stimulated for 1 hour, 2 hours, 4 hours, and 6 hours with atosiban $(10 \mu \mathrm{M})$ and/or OT $(10 \mathrm{nM})$. (A-E) The expression of downstream NF- $\kappa$ B-regulated genes, COX-2 (A), IL-6 (B), CCL5 (C), IL-8 (D), and SOD2 (E), was examined from total RNA extracts. Atosiban inhibited the effect on OT-induced upregulation of COX-2, IL-6, and IL- 8 but failed to inhibit CCL5 expression and it alone increased the expression of CCL5 $(n=6 ; * P<$
Treatment of amnion cells with nolasiban also completely inhibited OT-mediated expression of PG synthetic enzymes COX-2 and p-cPLA ${ }_{2}$ from the lowest dose of $1 \mu \mathrm{M}(P<0.05$ and $P<0.001$, respectively; Fig. 5) and, consequently, OT-mediated $\mathrm{PGE}_{2}$ release $(P<0.001$; Fig. 6). Further experiments looking at the mRNA expression of other $\mathrm{NF}-\kappa \mathrm{B}-$ regulated genes revealed a clear dose-dependent inhibitory effect of nolasiban on COX-2, IL-6, CCL5, IL-8, CCL2, and superoxide dismutase 2 (Fig. 7).

Atosiban Acts as a Partial Inhibitor of OT-Mediated Responses, But It Alone Consistently Activates NF- $\mathrm{B}$ and ERK1/2, While Inducing CCL5 Expression in Human Myometrium. In contrast with our findings in human amnion, treatment with atosiban significantly reduced the OT-driven activation of p38 kinase and the upregulation of COX-2 in human myometrial cells (Fig. 8). However, despite these inhibitory effects, atosiban failed to suppress the effect of OT on NF- $\kappa \mathrm{B}$ and ERK1/2 activation and resulted in activation of both $\mathrm{NF}-\kappa \mathrm{B}$ and ERK1/2 in the absence of OT (Fig. 8).

The presence of atosiban showed variable effects on the expression of downstream NF- $\kappa \mathrm{B}-$ regulated genes. Complementary to the findings in COX-2 expression at the protein level, atosiban successfully inhibited the OT-induced mRNA expression of COX-2 and IL-8 (Fig. 9, A and D). There was a marginal reduction in OT-induced IL-6 expression, but it did not reach statistical significance (Fig. 9B). Of particular note were the changes in the mRNA expression of CCL5, which increased with atosiban in the absence of OT stimulation (Fig. 9C).

Similar to Amnion, Nolasiban Suppresses the Effects of OT on Proinflammatory Mediators in Human Myometrium. Myometrial smooth muscle cells were incubated with increasing doses of nolasiban with or without OT stimulation. The OTR antagonist (nolasiban) alone had no significant effect on the OT-induced activation of NF- $\kappa \mathrm{B}$ and MAPKs, and the presence of nolasiban blocked the effects of OT on NF- $\kappa \mathrm{B}$ and p38 kinase, reaching significance at the $10-\mu \mathrm{M}$ dose $(P<0.01$; Fig. 10). Moreover, nolasiban suppressed the OT-driven upregulation of COX-2 and p-cPLA ${ }_{2}$ (Fig. 11), ultimately leading to the reduction of $\mathrm{PGE}_{2}$ synthesis (Fig. 12). In addition to COX-2 expression, nolasiban also resulted in suppression of other NF- $\kappa$ Bregulated genes, IL-8, IL-6, and CCL5, in a dose-dependent manner (Fig. 13, A-D). Similar to amnion, nolasiban treatment alone in the myometrium had no effect on activation of NF- $\kappa \mathrm{B}$ and p38 or expression of PG synthetic enzymes (Supplemental Fig. 2). At the 30- $\mu \mathrm{M}$ dose, nolasiban activated ERK1/2 $(P<0.01)$ without affecting the expression of downstream targets such as COX-2 and $\mathrm{p}-\mathrm{cPLA} \mathrm{A}_{2}$.

\section{Discussion}

Here, we investigated the effect of nolasiban, a novel orally active OTR antagonist, on OT-driven activation of inflammatory responses in human gestational tissues. We previously

0.05 versus NS; ${ }^{\#} P<0.05$ versus OT-treated samples; ANOVA). Significance was tested using ANOVA, with correction for multiple testing by the Tukey-Kramer multiple comparisons test. ANOVA, analysis of variance; Ato, atosiban; GAPDH, glyceraldehyde 3-phosphate dehydrogenase; NS, nonstimulated; SOD, superoxide dismutase. 
A

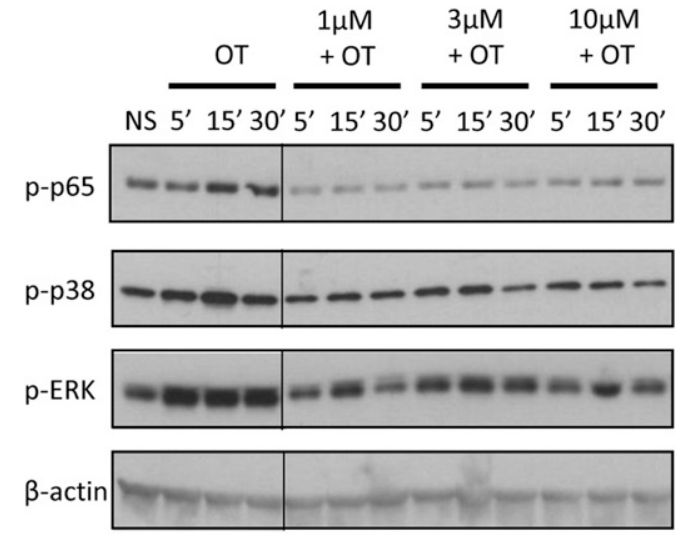

B
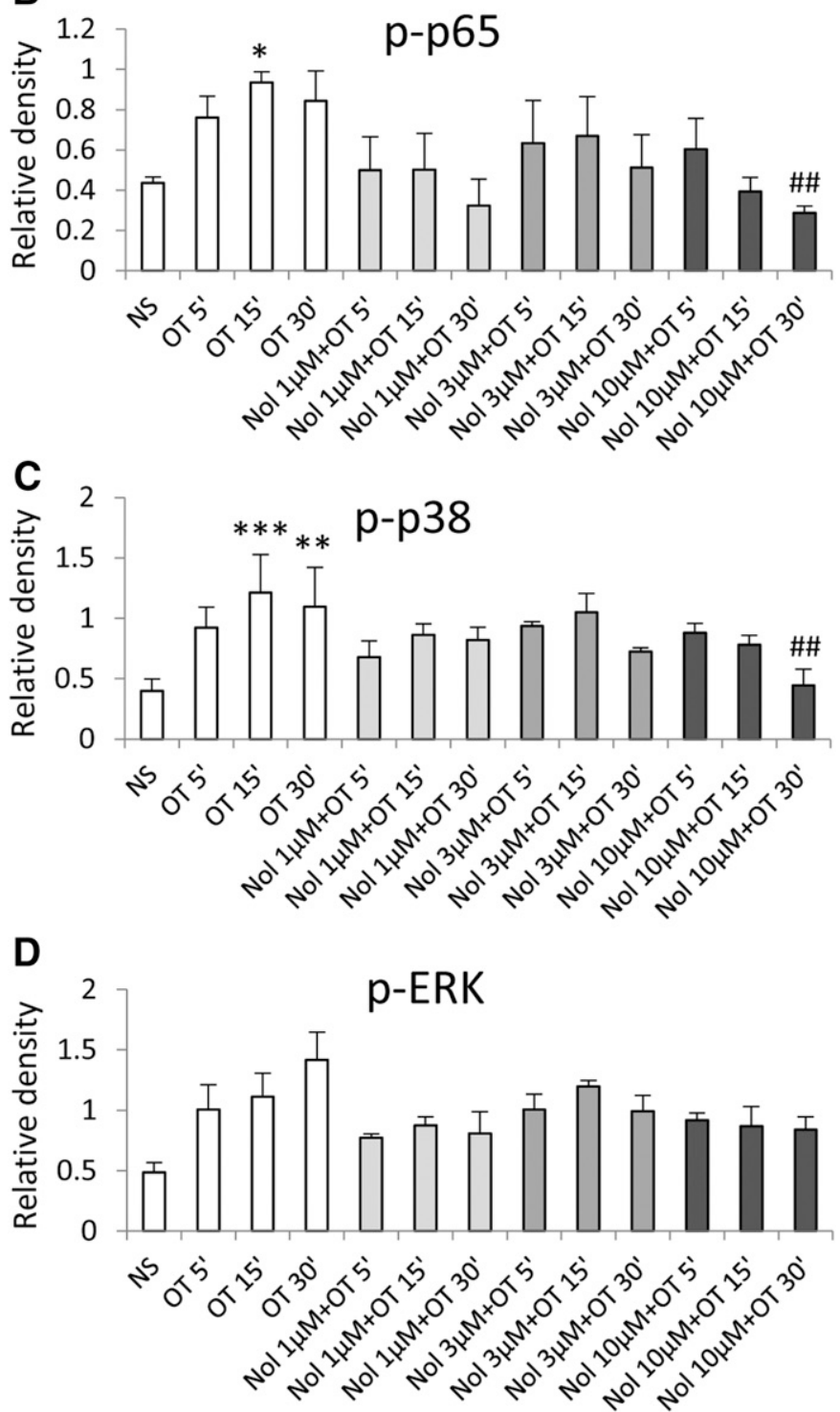

Fig. 10. The effect of nolasiban on OT-mediated activation of NF- $\kappa$ B and MAPKs in myometrial cells. Prelabor primary myometrial smooth muscle cells were stimulated with OT $(10 \mathrm{nM})$ in the presence of absence of nolasiban $(1,3$, or $10 \mu \mathrm{M})$ for 5 minutes, 15 minutes, and 30 minutes. (A) Western blots were used to examine the effect of nolasiban on OT-mediated activation of p65 NF- $\kappa$ B and MAPKs, ERK, and p38. NS controls and OT-only treatments were adjusted to contain the vehicle, DMSO, at $0.1 \%(\mathrm{v} / \mathrm{v})$. $\beta$-actin confirmed equal protein loading. (B-D) Densitometric
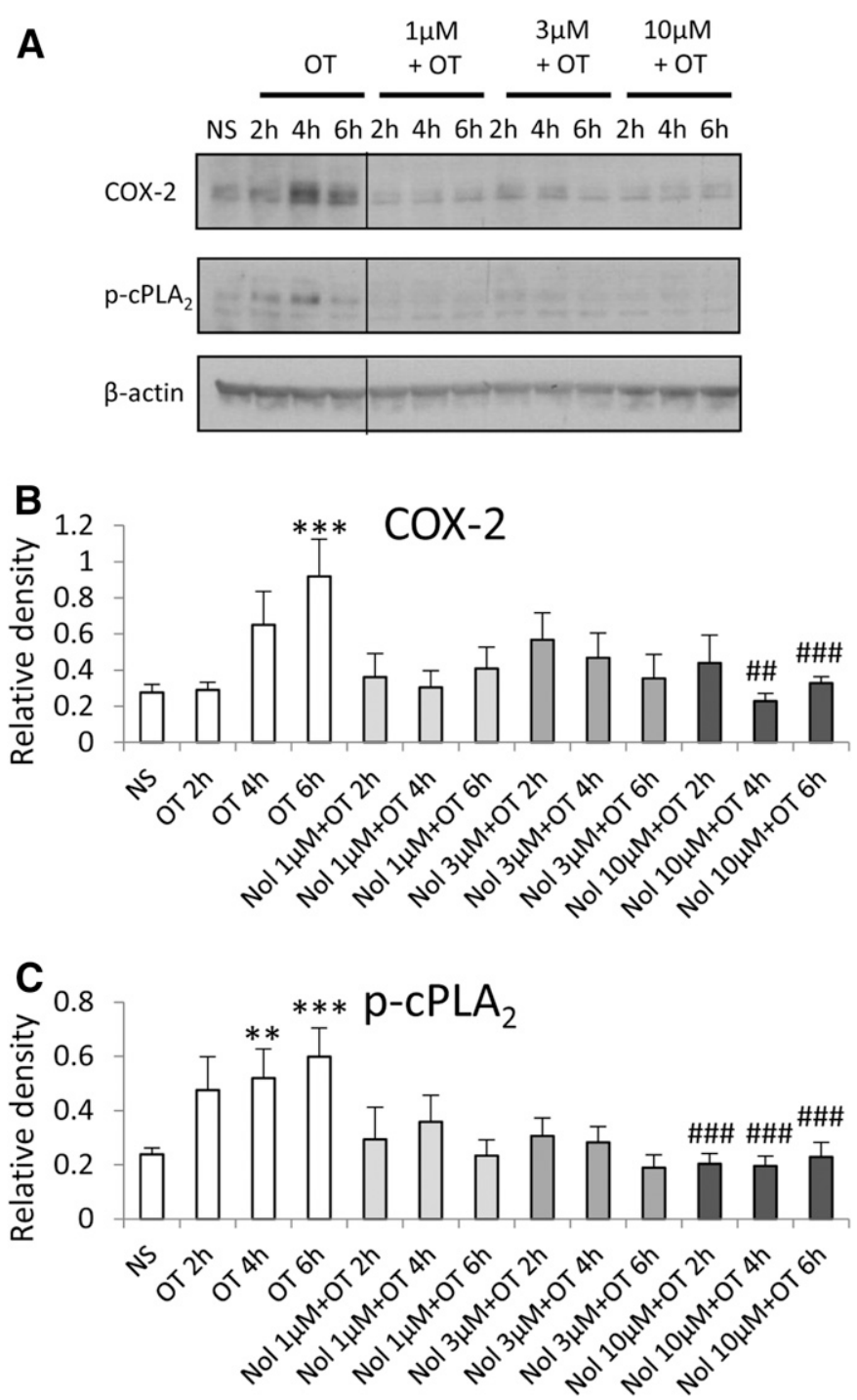

Fig. 11. The effect of nolasiban on OT-mediated expression of PG synthetic enzymes in myometrial cells. Prelabor primary myometrial smooth muscle cells were stimulated with OT $(10 \mathrm{nM})$ in the presence or absence of nolasiban (1, 3, or $10 \mu \mathrm{M}$ ) for 2 hours, 4 hours, and 6 hours. (A) Western blots were used to examine the effect of nolasiban on OT-mediated expression of COX-2 and p-cPLA 2 . NS controls and OT-only treatments were adjusted to contain the vehicle, DMSO, at $0.1 \%(\mathrm{v} / \mathrm{v}) . \beta$-actin confirmed equal protein loading. (B and C) Densitometric analyses showed significant decreases in OT-mediated expression of COX-2 (B) and p-cPLA (C) in the presence of nolasiban $(n=6$; **P $<0.01$; *** $P<0.001$ versus NS; ${ }^{\# \#} P<0.01$; ${ }^{\# \# \# P}<0.001$ versus OT-treated samples; ANOVA). Significance was tested using ANOVA, with correction for multiple testing by the Tukey-Kramer multiple comparisons test. ANOVA, analysis of variance; Nol, nolasiban; NS, nonstimulated.

showed that OT activates a cassette of inflammatory mediators in both human amnion and myometrium, demonstrating a role for OT as an endogenous inflammatory signaling molecule (Kim et al., 2015). Atosiban, an OTR

analyses showed significant decreases in OT-mediated activation of p65 (B) and p38 (C) in the presence of nolasiban, but not ERK1/2 (D) $(n=6 ; * P<$ $0.05 ; * * P<0.01 ; * * * P<0.001$ versus NS; ${ }^{* \#} P<0.01$ versus OT-treated samples; ANOVA). Significance was tested using ANOVA, with correction for multiple testing by the Tukey-Kramer multiple comparisons test. ANOVA, analysis of variance; Nol, nolasiban; NS, nonstimulated. 


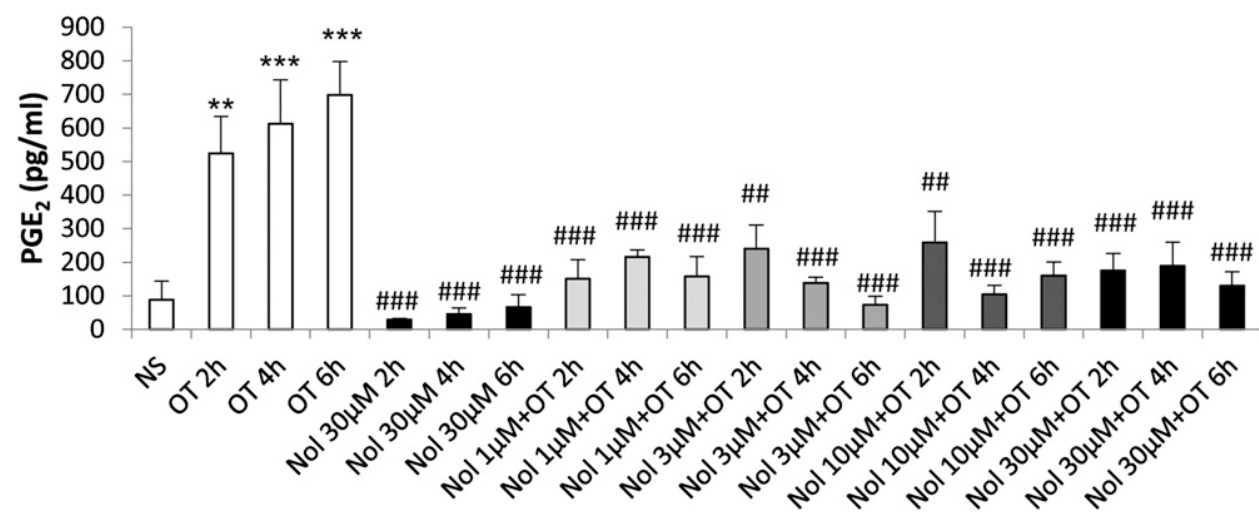

Fig. 12. The effect of nolasiban on OT-mediated $\mathrm{PGE}_{2}$ release in myometrial cells. Prelabor primary myometrial smooth muscle cells were stimulated with OT $(10 \mathrm{nM})$ in the presence or absence of nolasiban $(1,3,10$, or $30 \mu \mathrm{M})$ for 2 hours, 4 hours, and 6 hours. The $\mathrm{PGE}_{2}$ enzymelinked immunosorbent assay demonstrated a significant inhibition of OT-mediated $\mathrm{PGE}_{2}$ production by nolasiban $(n=6$; $* * P<0.01 ; * * * P<0.001$ versus NS; ${ }^{\# \# P} P<0.01 ;{ }^{\# \# \# P} P<0.001$ versus OT-treated samples; ANOVA). Significance was tested using ANOVA, with correction for multiple testing by the Dunn multiple comparisons test. ANOVA, analysis of variance; Nol, nolasiban; NS, nonstimulated. antagonist, is used in clinical practice in Europe for the treatment of preterm labor, and other OTR antagonists are in clinical trials. Although OTR antagonists can inhibit myometrial contractility, they appear to have differential effects upon mediators of inflammation within the gestational tissues. The OTR antagonist atosiban was shown previously to inhibit myometrial contractility (Phaneuf et al., 1994); however, atosiban only acts as a partial inhibitor of OT-mediated proinflammatory responses in human myometrium and has a purely stimulatory effect and no inhibitory effect in human amnion. We have shown that in contrast with atosiban, nolasiban suppresses OT-mediated proinflammatory effects in both human amnion and myometrium (Fig. 14).

We previously showed that there are differences in the OT-mediated activation of $\mathrm{NF}-\kappa \mathrm{B}$ between the amnion and
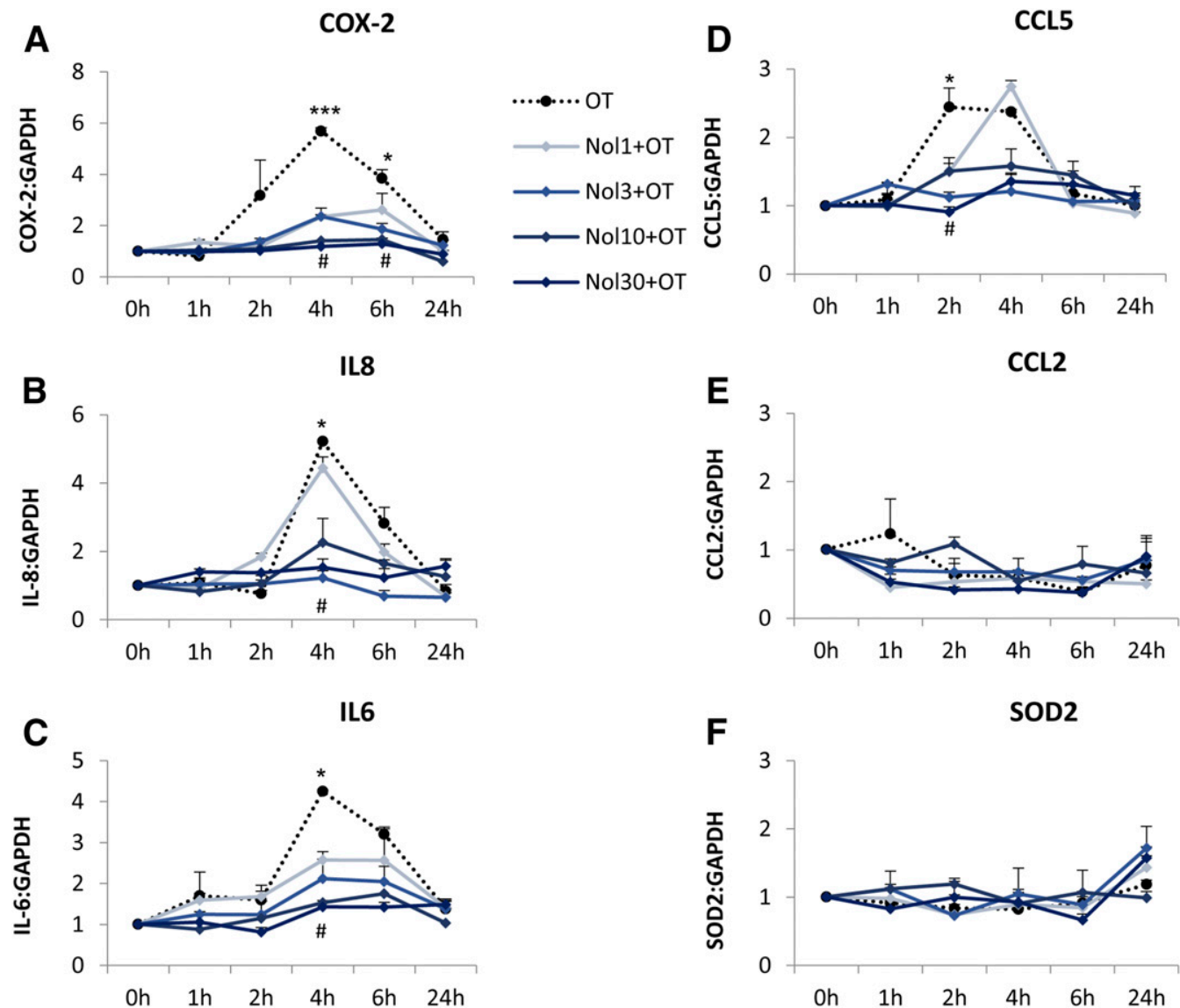

Fig. 13. The effect of nolasiban on OT-mediated expression of downstream NF- $\kappa$ B-regulated genes in myometrial cells. Prelabor primary myometrial smooth muscle cells were treated with OT (10 nM) in the presence or absence of nolasiban $(1,3,10$, or $30 \mu \mathrm{M})$ for 1 hour, 2 hours, 4 hours, 6 hours, and 24 hours, and the expression of downstream NF- $\kappa$ B-regulated genes, COX-2 (A), IL-8 (B), IL-6 (C), CCL5 (D), CCL2 (E), and SOD2 (F), was analyzed using qRT-PCR. For qRT-PCR analysis, target gene mRNA levels were normalized to that of the housekeeping gene, GAPDH $(n=4$; $* P<0.05 ; * * * P<0.001$ versus NS; ${ }^{P} P<0.05$ versus OT-treated samples; ANOVA). Significance was tested using ANOVA, with correction for multiple testing by the TukeyKramer multiple comparisons test. GAPDH, glyceraldehyde 3-phosphate dehydrogenase; Nol, nolasiban; NS, nonstimulated; qRT-PCR, quantitative real-time polymerase chain reaction; SOD, superoxide dismutase. 


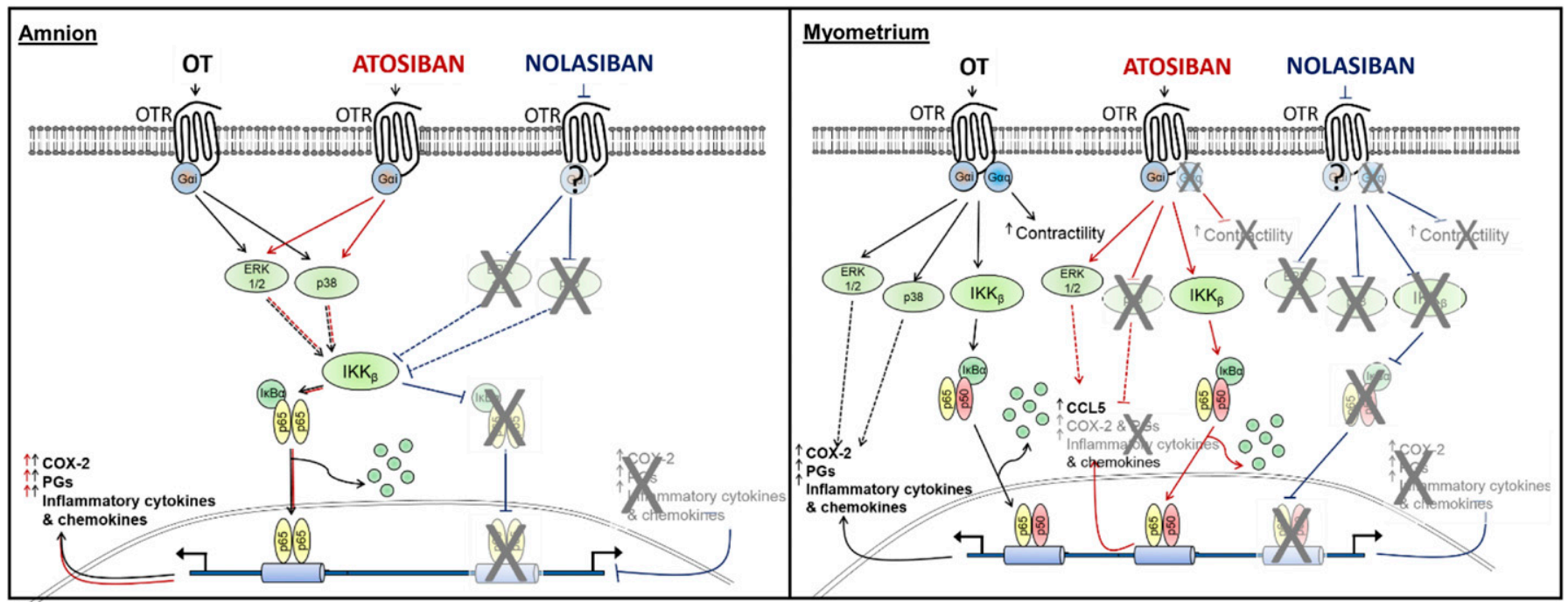

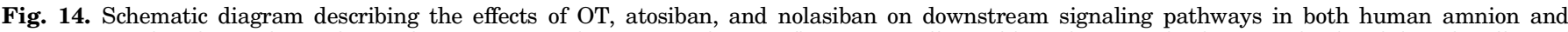

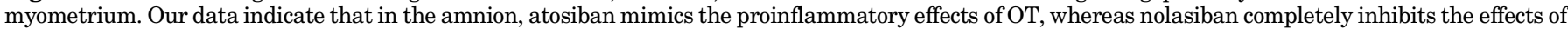

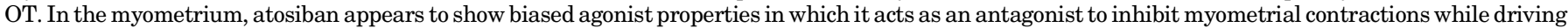
expression of the NF- $\mathrm{B}$-regulated gene, CCL5. Similar to amnion, nolasiban appeared to inhibit both the inflammatory and contractile effects of OT.

myometrium, which are associated with differential NF- $\kappa \mathrm{B}$ subunit translocation and MAPK crosstalk. In the myometrium, OT-driven NF- $\kappa \mathrm{B}$ activation resembles a canonical $\mathrm{NF}-\kappa \mathrm{B}$ signaling pathway involving both $\mathrm{p} 65$ and p50 translocation. OT-induced NF- $\kappa \mathrm{B}$ activation in the amnion involves nuclear translocation of $\mathrm{p} 65$ but not $\mathrm{p} 50$, and requires ERK1/2 and p38 activation (Kim et al., 2015). Such tissuespecific signaling may provide an explanation for the variable effects of atosiban in the amnion and myometrium: atosiban acts as an "antilabor" agent though inhibition of OT-induced contractility in the myometrium, whereas it acts as a "prolabor agent" in the amnion and partially so in the myometrium, possibly via differential OTR G-protein coupling in the two cell types. In the amnion, atosiban has no inhibitory effect and, notably, it alone mimics the proinflammatory effects of OT. In the myometrium, atosiban significantly reduces OT-driven activation of p38 kinase and upregulation of COX-2 and IL-8, but it remains unable to suppress the effect of OT on NF- $\kappa$ B and ERK1/2 activation and increases CCL5 expression regardless of OT stimulation. The data presented here show that, unlike atosiban, nolasiban completely inhibits all OT-mediated responses and suppresses expression and activation of proinflammatory mediators both in the amnion and myometrium without any downstream effects when treated alone. Despite the complete inhibition of OT-induced COX-2 upregulation and $\mathrm{PGE}_{2}$ levels in the presence of nolasiban, relatively high levels of $\mathrm{PGE}_{2}$ were detected in samples treated with nolasiban and OT at earlier time points. This may be due to the release of $\mathrm{PGE}_{2}$ rather than synthesis, as nolasiban successfully inhibits COX-2 expression as early as 2 hours. There have been reports in rat models in which OT was able to stimulate immediate pulsatile release of $\mathrm{PGE}_{2}$ via $\mathrm{Ca}^{2+}$ responses (Chen et al., 2015).

Preterm and term labor are characterized by a marked inflammatory cascade (Osman et al., 2003; Shynlova et al., 2013) associated with the upregulation of proinflammatory cytokines and chemokines, such as IL- $1 \beta$, IL-6, IL-8, CCL2, and CCL5, in both human amnion and myometrium. The amnion is an important site of PG and proinflammatory cytokine/chemokine release and leads to fetal membrane remodeling and cervical ripening (Kim et al., 2015). Fetal membrane activation is considered to be a prelude to the onset of labor (Lim et al., 2012). This involves activation of inducible $\mathrm{NF}-\kappa \mathrm{B}$ and the upregulation of prolabor proinflammatory genes such as COX-2 and OTR (Lim et al., 2012). We previously demonstrated that treatment with OT drives a similar set of inflammatory gene expressions as those in amnion activation, indicating that OT may act as an endogenous inflammatory signaling molecule in human gestational tissues (Kim et al., 2015).

Knowledge of GPCRs continues to evolve, and the diversity of its actions and complexity of its signaling mechanisms are becoming increasingly evident (Berchiche and Sakmar, 2016). The biology of the OT/OTR system appears to be no exception. Nolasiban is a novel orally active, small-molecule, nonpeptide OTR antagonist that is more selective for OTR than atosiban, as it binds to the human OTR with a $K_{\mathrm{i}}$ of $52 \mathrm{nM}$ and is 2.5- and 50-fold selective against AVP V1a and V2 receptors, respectively. We previously showed that nolasiban can dose-dependently inhibit spontaneous and OT-stimulated contractions, affecting the rate, contraction peak, tension, and contraction duration to a similar extent as atosiban (Arulkumaran et al., 2016). Unlike atosiban, inhibition of uterine contractions by nolasiban did not evoke a proinflammatory signaling pathway; therefore, nolasiban appears to be a pure OTR antagonist, blocking both $G_{\alpha i^{-}}$and $G_{\alpha q^{-}}$-mediated OT signaling in both the amnion and myometrium.

Early preterm birth, typically classified as delivery before 34 weeks of gestation, is often associated with infection and/or inflammation. It is now recognized that cytokinemediated cerebral injury represents an important cause of prematurity-linked complications; thus, inflammation within the uterus is an important risk factor for fetal and neonatal central nervous system damage and poor outcomes 
(Peebles, 2007). Therefore, it is crucial that any tocolytic agent used in acute preterm labor, including those modulating the OT/OTR system, should evade activation or aggravation of inflammation. Our study indicates that nolasiban is a promising new tocolytic, as it has the ability to not only inhibit myometrial contractility but it can also suppress the OT-mediated proinflammatory responses in human gestational tissues.

\section{Acknowledgments}

The authors thank the labor ward patients and staff at Queen Charlotte's and Chelsea Hospital for provision of and assistance with the collection of placenta and myometrial biopsies.

\section{Authorship Contributions}

Participated in research design: Kim, Bennett, Terzidou.

Conducted experiments: Kim, Fairhurst.

Contributed new reagents or analytic tools: Pohl, Chollet, Gotteland.

Performed data analysis: Kim, Bennett, Terzidou.

Wrote or contributed to the writing of the manuscript: Kim, Pohl, Chollet, Gotteland, Bennett, Terzidou.

\section{References}

Arulkumaran S, Kim SH, Pohl O, Chollet A, Bennett PR, and Terzidou V (2016) The OTR antagonist, OBE001, inhibits both spontaneous and OT-induced contractions of human pregnant myometrium in vitro (Abstract). Reprod Sci 23 (Suppl 1):299A.

Bennett PR, Rose MP, Myatt L, and Elder MG (1987) Preterm labor: stimulation of arachidonic acid metabolism in human amnion cells by bacterial products. Am J Obstet Gynecol 156:649-655.

Berchiche YA and Sakmar TP (2016) CXC chemokine receptor 3 alternative splice variants selectively activate different signaling pathways. Mol Pharmacol 90: 483-495.

Blanks AM and Thornton S (2003) The role of oxytocin in parturition. BJOG 110 (Suppl 20):46-51

Busnelli M, Saulière A, Manning M, Bouvier M, Galés C, and Chini B (2012) Functional selective oxytocin-derived agonists discriminate between individual G protein family subtypes. $J$ Biol Chem 287:3617-3629.

Chen D, Zhao J, Wang H, An N, Zhou Y, Fan J, Luo J, Su W, Liu C, and Li J (2015) Oxytocin evokes a pulsatile PGE2 release from ileum mucosa and is required for repair of intestinal epithelium after injury. Sci Rep 5:11731.

Chollet A (2015) inventor, ObsEva SA, assignee. Pyrrolidine derivatives as oxytocin/vasopressin V1a receptors antagonists. World Intellectual Property Organization patent WO/2015/036160. 2015 Mar 19.

Dommergues MA, Patkai J, Renauld JC, Evrard P, and Gressens P (2000) Proinflammatory cytokines and interleukin-9 exacerbate excitotoxic lesions of the newborn murine neopallium. Ann Neurol 47:54-63.
Grether JK and Nelson KB (1997) Maternal infection and cerebral palsy in infants of normal birth weight. JAMA 278:207-211.

Haas DM, Caldwell DM, Kirkpatrick P, McIntosh JJ, and Welton NJ (2012) Tocolytic therapy for preterm delivery: systematic review and network meta-analysis. BMJ 345:e6226.

Howson CP, Kinney MV, McDougall L, and Lawn JE; Born Too Soon Preterm Birth Action Group (2013) Born too soon: preterm birth matters. Reprod Health 10 (Suppl 1):S1.

Kim SH, MacIntyre DA, Firmino Da Silva M, Blanks AM, Lee YS, Thornton S, Bennett PR, and Terzidou V (2015) Oxytocin activates NF-kB-mediated inflammatory pathways in human gestational tissues. Mol Cell Endocrinol 403: 64-77.

Kim SH, MacIntyre DA, Hanyaloglu AC, Blanks AM, Thornton S, Bennett PR, and Terzidou V (2016) The oxytocin receptor antagonist, Atosiban, activates proinflammatory pathways in human amnion via G( $\alpha$ i) signalling. Mol Cell Endocrinol 420:11-23.

Lawn JE, Davidge R, Paul VK, von Xylander S, de Graft Johnson J, Costello A, Kinney MV, Segre J, and Molyneux L (2013) Born too soon: care for the preterm baby. Reprod Health 10 (Suppl 1):S5.

Lim S, MacIntyre DA, Lee YS, Khanjani S, Terzidou V, Teoh TG, and Bennett PR (2012) Nuclear factor kappa B activation occurs in the amnion prior to labour onset and modulates the expression of numerous labour associated genes. PLoS One 7: e34707.

Manning M, Stoev S, Cheng LL, Wo NC, and Chan WY (2001) Design of oxytocin antagonists, which are more selective than atosiban. J Pept Sci 7:449-465.

Osman I, Young A, Ledingham MA, Thomson AJ, Jordan F, Greer IA, and Norman JE (2003) Leukocyte density and pro-inflammatory cytokine expression in human fetal membranes, decidua, cervix and myometrium before and during labour at term. Mol Hum Reprod 9:41-45.

Peebles DM (2007) Intrauterine Infection and Perinatal Brain Injury (Scientific Impact Paper No. 3), Royal College of Obstetricians and Gynaecologists, London.

Phaneuf S, Asbóth G, MacKenzie IZ, Melin P, and López Bernal A (1994) Effect of oxytocin antagonists on the activation of human myometrium in vitro: atosiban prevents oxytocin-induced desensitization. Am J Obstet Gynecol 171: 1627-1634.

Phaneuf S, Europe-Finner GN, Varney M, MacKenzie IZ, Watson SP, and López Bernal A (1993) Oxytocin-stimulated phosphoinositide hydrolysis in human myometrial cells: involvement of pertussis toxin-sensitive and -insensitive G-proteins. $J$ Endocrinol 136:497-509.

Shynlova O, Lee YH, Srikhajon K, and Lye SJ (2013) Physiologic uterine inflammation and labor onset: integration of endocrine and mechanical signals. Reprod Sci 20:154-167.

Thornton C, Rousset CI, Kichev A, Miyakuni Y, Vontell R, Baburamani AA, Fleiss B, Gressens P, and Hagberg H (2012) Molecular mechanisms of neonatal brain injury. Neurol Res Int 2012:506320.

Whalen EJ, Rajagopal S, and Lefkowitz RJ (2011) Therapeutic potential of $\beta$-arrestin- and G protein-biased agonists. Trends Mol Med 17:126-139.

Wu YW and Colford JM, Jr (2000) Chorioamnionitis as a risk factor for cerebral palsy: a meta-analysis. JAMA 284:1417-1424.

Address correspondence to: Dr. Vasso Terzidou, Institute of Reproductive and Developmental Biology, Hammersmith Hospital Campus, Imperial College London, Du Cane Road, London W12 0NN, UK. E-mail: v.terzidou@ imperial.ac.uk 